\title{
Self-Assembled Amphiphilic Molecules for Highly Efficient Photocatalytic Hydrogen Evolution from Water
}

Hyun-Jun Lee , ${ }^{1}$ Jaekwan Kim, ${ }^{1}$ Abasi Abudulimu, ${ }^{2}$ Juan Cabanillas-Gonzalez, ${ }^{2}$ Paramjyothi C. Nandajan, ${ }^{2}$ Johannes Gierschner, ${ }^{* 2}$ Larry Lüer, ${ }^{* 2}$ Soo Young Park ${ }^{*, 14}$

'Laboratory of Supramolecular Optoelectronic Materials, Department of Material Science and Engineering, Seoul National University, o8826 Seoul, Korea.

IMDEA Nanoscience, Calle Faraday 9, Campus Cantoblanco, 28049 Madrid, Spain.

Institute of Materials for Electronics and Energy Technology (i-MEET), Friedrich-Alexander University Erlangen-Nürnberg, Martensstraße 7, 91058 Erlangen, Germany

4Research Institute of Advanced Materials (RIAM), Seoul National Univeresity, o8826 Seoul, Korea.

Supporting materials 


\section{Experimental Procedures}

\section{Materials}

Molecule 1 was synthesized according to the reported method. ${ }^{1}$ Since palladium catalyst was used in its synthetic route, we analyze amount of the metal in the final product through ICP-AES. No remaining palladium was observed (detection limit $<$ few $p p b$ ). All other chemicals were purchased from Sigma-Aldrich, Acros and Alfa Aesar, and used without further purification.

\section{Methods - General}

UV-Vis absorption spectra were measured using a Shimazu, UV-1650 PC spectrometer. Dynamic Light Scattering (DLS) measurements were performed on an Otsuka Electronics, DLS-7000. Electrophoretic light scattering (ELS) measurements were conducted by using an ELS Z-1000. Transmission electron microscope (TEM) images, scanning transmission microscope (STEM) images, high resolution transmission microscope (HRTEM) images and energy dispersive spectrometry (EDS) were recorded on a JEOL, JEM-2100F and JEM-3010, JEM-F200 and FEI, Tecnai F20. The TEM samples were prepared by dropping a droplet of each aqueous solution on a copper grid and drying in ambient condition. The cyclic voltammetry experiments were conducted using a Princeton Applied Research Potentiostat/Galvanostat Model 273A (Princeton Applied Research) equipped with a three electrode cell assemble including an ITO-coated glass working electrode, a platinum wire counter electrode, and a silver wire quasi-reference electrode. The HOMO level was calculated using the onset oxidation potential $\left(\mathrm{E}_{\mathrm{ox}}\right)$, and ferrocene $\left(\mathrm{E}_{\mathrm{Fc} / \mathrm{Fc}+}\right.$ reference was used for calibration. $\left(\mathrm{E}_{\mathrm{HOMO}}=-\left[\mathrm{E}_{\mathrm{Ox}}-\mathrm{E}_{\mathrm{FC} / \mathrm{Fc}+}+4.8\right] \mathrm{eV}\right)$. The LUMO level was calculated from the electrochemically measured HOMO level and the band gap derived from UV-Vis absorption edge. $\left(E_{\text {LUMO }}=E_{\text {HOMO }}+E g\right)$ ICP-AES was conducted using Perkin-EImer, OPTIMA 4300DV. The apparent quantum yield (AQY) calculated by using a $300 \mathrm{~W}$ Xe lamp equipped with a Newport 20BPF70-550 band-pass filter. (AQY $=[2 \times$ the number of evolved hydrogen molecules in an hour] / [the number of incident photons in an hour]) All photons passing through the filter were assumed to have an energy of $550 \mathrm{~nm}$. The sample for AQY measurement was prepared from $0.5 \mu \mathrm{mol}$ of $\mathrm{K}_{2} \mathrm{PtCl}_{4}, 0.65 \mu \mathrm{mol}$ of molecule $1,0.65 \mu \mathrm{mol}$ of Erythrosin B, $12 \mathrm{mmol}$ of L-ascorbic acid and $2 \mathrm{mmol}$ of Nal.

\section{Methods - Hydrogen Evolution Experiment (for each sample)}

\section{3-1 Self-assembled molecules 1 (SM1)}

The Sample for photocatalytic hydrogen evolution were prepared by adding $0.65 \mathrm{~mL}$ of 1 $\mathrm{mM}$ aqueous solution of molecule 1 into $0.97 \mathrm{M} 12.35 \mathrm{~mL}$ of aqueous solution of L-ascorbic acid. Before mixing $\mathrm{pH}$ of ascorbic acid solution was adjusted as 4 by adding $2 \mathrm{~N} \mathrm{NaOH}(\mathrm{aq})$ solution. To maximize Total $13 \mathrm{~mL}$ of aqueous solution (final molar concentration of ascorbic acid $\sim 0.92 \mathrm{M}$ ) was added into a $40 \mathrm{~mL}$ air-tight vial equipped with a Teflon septum. The sample in the light-shielded vial was bubbled for 15 min by Ar with $1 \% \mathrm{CH}_{4}$ as internal standard. Then the vial was irradiated with a $300 \mathrm{~W}$ Xe lamp with a $400 \mathrm{~nm}$ cutoff filter to eliminate ultraviolet light. The gas $(200 \mu \mathrm{L})$ in the headspace above the sample was extracted using a Hamilton Luerlock type syringe then injected into a gas chromatography (Agilent $7890 \mathrm{~A}$ ) equipped with metal packed GC column (60/80 molecular sieve 5A support). The amount of hydrogen evolved was calculated with the integrated area of hydrogen signal calibrated by the integrated area of $\mathrm{CH}_{4}$ signal.

3-2 SM1 with iodide (SM1/I) 
The measurement was carried out under the same conditions as a (SM1) except that sodium iodide was added ( $0.15 \mathrm{M}$ in final sample).

3-3 SM1 without iodide with platinum

The sample was prepared according to the same procedures as a (SM1) except that $0.5 \mathrm{~mL}$ of $1 \mathrm{mM} \mathrm{K}_{2} \mathrm{PtCl}_{4}$ aqueous solution was added. Volume of final sample was $13 \mathrm{~mL}$. The sample was added into a $40 \mathrm{~mL}$ air-tight vial equipped with a Teflon septum. It was continuously purged by 5 sccm of Ar. When TOF was measured every 30 minutes of visible light irradiation, the gas flew into a column of GC for few seconds and the amount of $\mathrm{H}_{2}$ evolved within the gas during that time was calculated by thermal conductivity detector (TCD). The exact amount of hydrogen was determined by the calibration value which was obtained by measuring calibration gas $\left(0.1 \mathrm{~mol} \%\right.$ of $\mathrm{H}_{2}$ in $\left.\mathrm{Ar}\right)$. TOF at $\mathrm{t}=0$ min was calculated by extrapolating TOF at $\mathrm{t}=30 \mathrm{mins}$ and TOF at $\mathrm{t}=60 \mathrm{mins}$. The amount of hydrogen evolved (or TON) vs. time curve was drawn by integrating the $\mathrm{H}_{2}$ evolution rate (or TOF) vs. time curve, $\mathrm{TON}_{\mathrm{Pt}}=\mathrm{n}\left(\mathrm{H}_{2}\right) / \mathrm{n}(\mathrm{Pt})$ or $\mathrm{TON}_{1}=\mathrm{n}\left(\mathrm{H}_{2}\right) / \mathrm{n}(\mathbf{1})$

3-4 SM1 with iodide (SM1/I) and platinum

The sample was prepared according to the same procedures as 4-2 (SM1 with iodide) except that $0.5 \mathrm{~mL}$ of $1 \mathrm{mM} \mathrm{K}_{2} \mathrm{PtCl}_{4}$ aqueous solution was added. Volume of final sample was $13 \mathrm{~mL}$. For the $\mathrm{H}_{2}$ evolution measurement, the same method as $\mathrm{C}$ was used for this sample.

3-5Self-assembled ionic complex (SM1:E)

$0.65 \mathrm{~mL}$ of $1 \mathrm{mM}$ aqueous solution of molecule 1 and $0.65 \mathrm{~mL}$ of $1 \mathrm{mM}$ aqueous solution of erythrosine $B(E)$ was added into $11.7 \mathrm{~mL}$ of ascorbic acid aqueous solution. Final solution has $0.92 \mathrm{M}$ of L-ascorbic acid, $13 \mathrm{~mL}$ of volume. Its $\mathrm{pH}$ was adjusted as 4 . For the $\mathrm{H}_{2}$ evolution measurement, the same method as $A$ was used for this sample.

3-6 SM1:E with iodide (SM1:E/I)

One sample was prepared according to the same procedures as e except that sodium iodide was added $(0.15 \mathrm{M}$ in final sample). This sample showed fast HER $(13 \mu \mathrm{mol} / \mathrm{g} \cdot \mathrm{h})$ in early stage, but it was saturated soon. The other sample was prepared by adding $0.65 \mathrm{~mL}$ of 1 $\mathrm{mM}$ aqueous solution of molecule 1 and $0.65 \mathrm{~mL}$ of $1 \mathrm{mM}$ aqueous solution of $\mathbf{E}$ into $11.7 \mathrm{~mL}$ aqueous solution which had $1 \mathrm{~mL}$ of triethanolamine in it. Before mixing, its $\mathrm{pH}$ was adjusted as 7 by adding $1.8 \mathrm{~N} \mathrm{HCl}$ (aq) solution. The latter sample showed stable activity, and reached to $\mathrm{TON}_{1} \sim 0.5$. For the $\mathrm{H}_{2}$ evolution measurements, the same method as $A$ was used for these samples.

3-7SM1:E without iodide with platinum

The sample was prepared according to the same procedures as $4-5$ except that $0.5 \mathrm{~mL}$ of 1 $\mathrm{mM} \mathrm{K}_{2} \mathrm{PtCl}_{4}$ aqueous solution was added. Volume of final sample was $13 \mathrm{~mL}$. For $\mathrm{H}_{2}$ evolution measurement, the same method as $C$ was used for this samples.

3-8SM1:E with iodide (SM1:E/I) and platinum

The sample was prepared according to the same procedures as 4-5 except that $0.5 \mathrm{~mL}$ of 1 $\mathrm{mM} \mathrm{K}_{2} \mathrm{PtCl}_{4}$ aqueous solution and sodium iodide (molar concentration in final solution $\sim 0.15$ $\mathrm{M}$ ) was added. Volume of final sample was $13 \mathrm{~mL}$. For $\mathrm{H}_{2}$ evolution measurement, the same method as $\mathrm{C}$ was used for this samples.

4. Methods: Singular value decomposition

Singular value decomposition (SVD) is a matrix decomposition technique that reproduces the original matrix $A$ (which can be of any shape) by a product of 3 matrices $U, S$, and $V$ :

$$
A=U \times S \times V
$$

The most important features of SVD are:

- Both matrices $U$ and $V$ are unitary, which means that they consist of sets of orthonormal basis vectors (across columns and rows, respectively). 
- The diagonal matrix $S$ contains the singular values ordered by strength. This makes it possible to split the result matrices into two parts, considering only the strongest singular values as signal related (suffix "S") and all the rest as noise (suffix "N"):

$$
\begin{aligned}
& A_{\exp }=U_{s} \times S_{s} \times V_{s}+U_{N} \times S_{N} \times V_{N}, \operatorname{or}(2) \\
& A_{\exp }=U_{s} \times S_{s} \times V_{s}+N
\end{aligned}
$$

- The reduced set of signal related singular values in eq.3 is always optimal in the least squares sense, which means it solves the equation

$$
N=\min \left(\left(A_{\exp }-U_{s} \times S_{s} \times V_{s}\right)^{2}\right)
$$

This feature is very useful, because it allows us to perform a bias free global fit of our experimental TA spectrum $A_{\text {exp }}$. using a very fast and reliable method.

Our standard procedure is to start the analysis by performing an SVD and varying the number of signal related states until the residuals contain only noise. In doing so, we obtain an important initial characterization of our experiment:

- We know the minimum number of independent states necessary to describe the full TA spectrum at all times and probe energies.

- We reject the measurement noise right from the start and work only with the small matrices $U_{s}$ and $V_{s}$ for the rest of the fitting session, greatly reducing CPU cost and local minima problems in the fitting procedure.

Comparing eq.3

$$
A_{\text {exp }}=U_{s} \times S_{s} \times V_{s}+N,(3)
$$

with Lambert-Beer's law in matrix notation, $\boldsymbol{A}_{\exp }=\boldsymbol{\sigma} \times \boldsymbol{L c}+\boldsymbol{N}$, (with $\sigma$ the matrix of crosssections and Lc the matrix of area densities and $\mathrm{N}$ some measurement noise) it is tempting to associate $\mathbf{U}_{\mathbf{S}}$ with $\boldsymbol{\sigma}$, and $\mathbf{S}_{\mathbf{S}} \mathbf{X} \mathbf{V}_{\mathbf{S}}$ with $\mathbf{L} \mathbf{c}$, which would bring us immediately to the solution. Unfortunately however, this is only one of an infinite number of solutions with exactly the same quality.

In the list of SVD features above, we noted that both $U_{s}$ and $V_{s}$ consist of orthonormal basis vectors. The matrix Lc in eq. 3 is supposed to represent time-resolved concentrations of photoexcited states. As concentrations are always positive, the concentration-time vectors of the individual states can never be orthonormal amongst each other. The same is true for the cross-section spectra in the $\boldsymbol{\sigma}$ matrix. In a spectral range without SE or PB (and hence only positive values), it is impossible that the single cross-section spectra be mutually orthonormal.

The problem is that there is an infinite number of any matrices $\mathbf{p}$ and $\mathbf{q}$ whose matrix product reproduces $A_{\text {exp }}$. We see that if we insert the identity $\mathbf{R} \times \mathbf{R}^{-1}$ into eq.3:

$$
A_{\text {exp }}=\sigma \times R \times R^{-1} \times L c+N
$$


Any matrix $\mathbf{R}$ that can be inverted will produce the same optimal fit. The matrix $\mathbf{R}$ is called the rotation matrix; it is the key to solve the problem. Comparing eq. 3 with eq. 5 yields

and

$$
U_{s}=\sigma \times R(6)
$$

$$
L c=R \times S_{s} \times V_{s}(7)
$$

Equation 6 tells us that the single basis spectra in $U_{\mathbf{S}}$ can be understood as linear combinations of the real photophysical cross-section spectra in $\boldsymbol{\sigma}$. The rotation matrix $\mathbf{R}$ can then also be understood as the matrix of spectral weights $R_{i, j}$ of each physical crosssection spectrum $\sigma_{i}(\lambda)$ in each basis spectrum $\mathbf{U}_{\mathbf{s}, j}(\lambda)$.

Using our photophysical knowledge about the system, we can provide realistic test functions for $\sigma_{i}(\lambda)$ and thus find $\mathbf{R}$ by non-linear optimization (spectral modeling). Once we found $\mathbf{R}$, we can use it to obtain the desired photoexcitation dynamics in Lc in eq. 7.

In Figures S20 - S26, we apply this strategy to isolate photoexcitation dynamics for a multitude of excited states in the same experiment. This is accomplished by starting with a system of which the excited states are known; we can then re-use these excited states in fitting more complicated samples. 
(a)

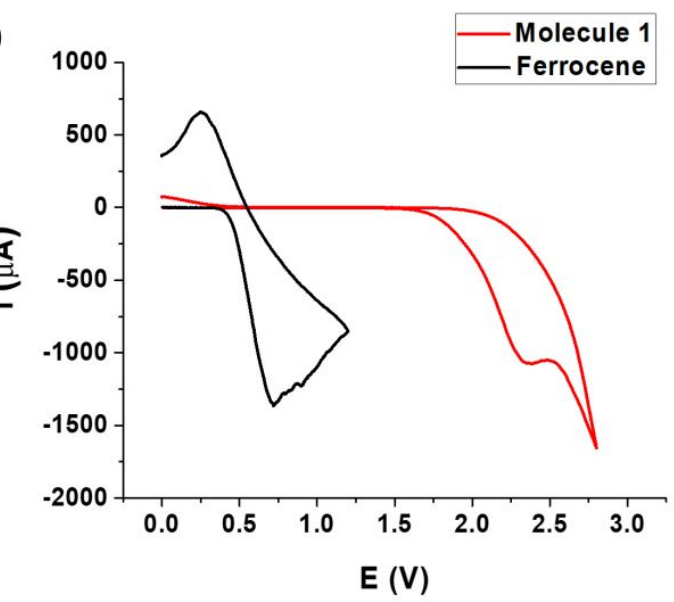

(c)

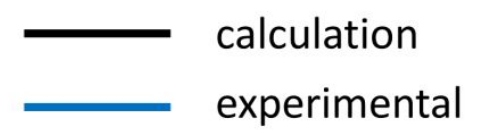

$-6.29 \mathrm{eV}$

$-3.30 \mathrm{eV}$
$-3.86 \mathrm{eV}$

$-6.70 \mathrm{eV}$

Figure S1. Cyclic voltammetry curves of (a) spin coated films on ITO of ferrocene and Molecule 1, (b) DMF solution of ferrocene and Erythrosin B, and (c) frontier orbital energy levels of Molecule 1 and Erythrosin B (b)
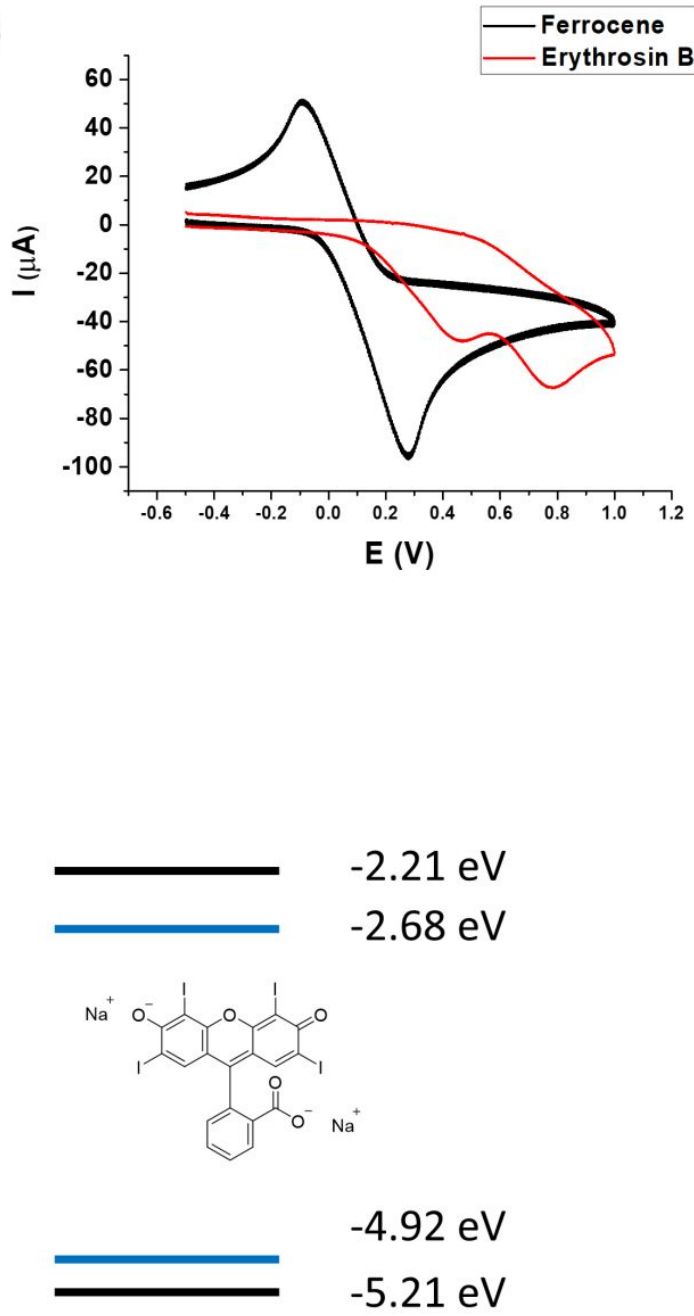
(a)

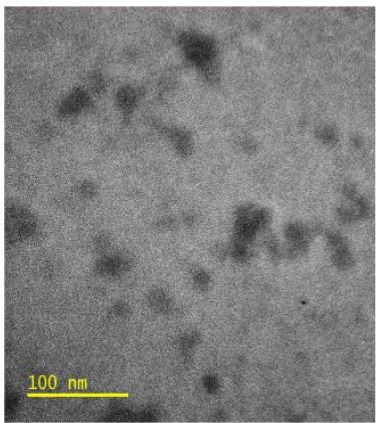

(c)

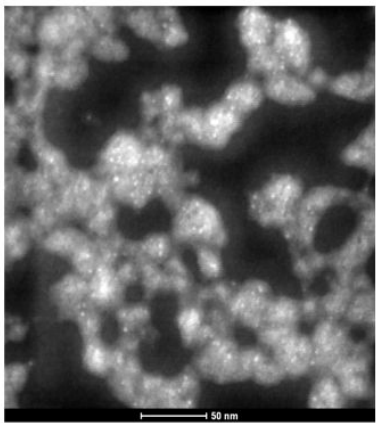

(e)

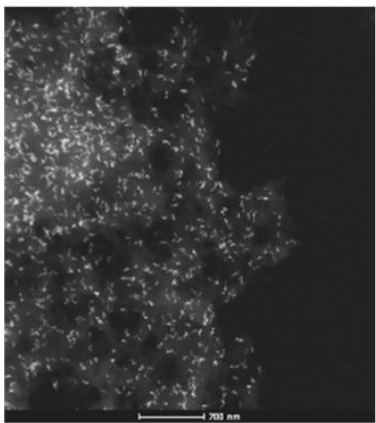

(b)

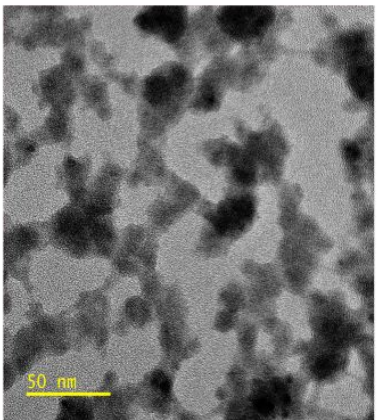

(d)
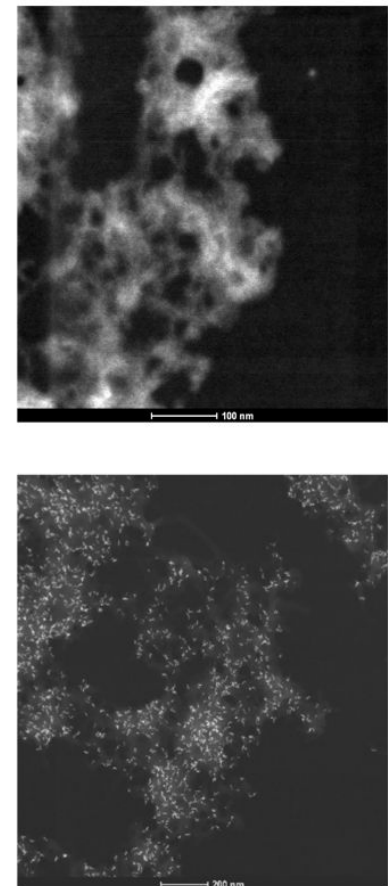

Figure S2. TEM images of (a) SM1 (prepared from $50 \mu \mathrm{M}$ aqueous solution of 1) (b) SM1 with iodide (prepared from $0.65 \mu \mathrm{mol}$ of 1 in $13 \mathrm{~mL}$ of $0.15 \mathrm{M}$ of Nal aqueous solution), STEM images of (c) SM1 with iodide (d) as prepared photocatalytic experimental sample (prepared from $0.5 \mu \mathrm{mol}$ of $\mathrm{K}_{2} \mathrm{PtCl}_{4}, 0.65 \mu \mathrm{mol}$ of 1 and $12 \mathrm{mmol}$ of L-ascorbic acid in $13 \mathrm{~mL}$ of $0.15 \mathrm{M}$ of $\mathrm{Nal})(\mathrm{e})$ after irradiated 24 hours 
(a)

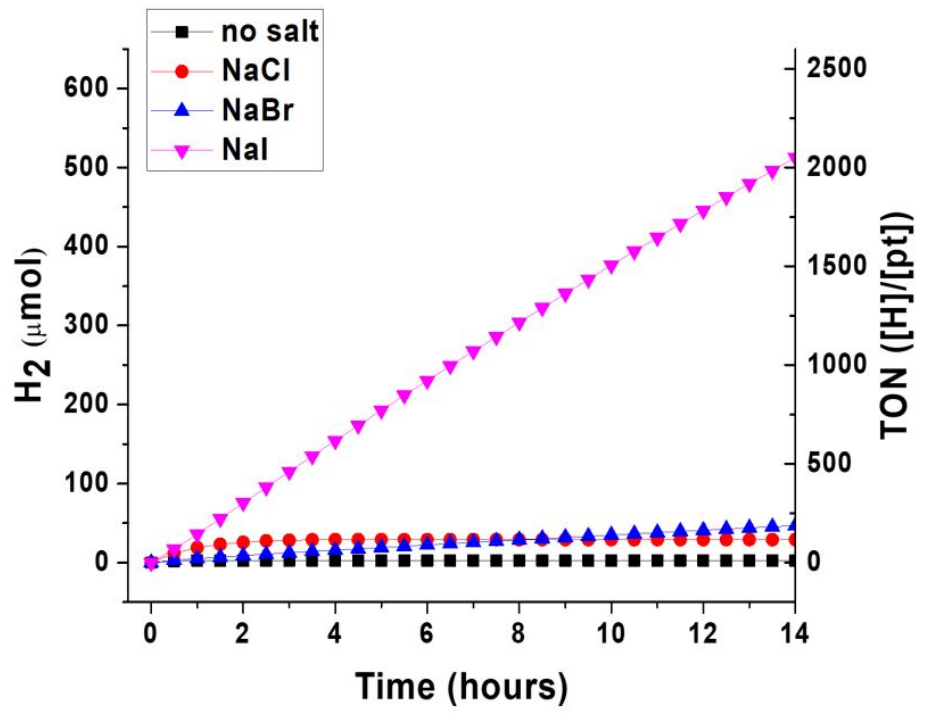

(b)

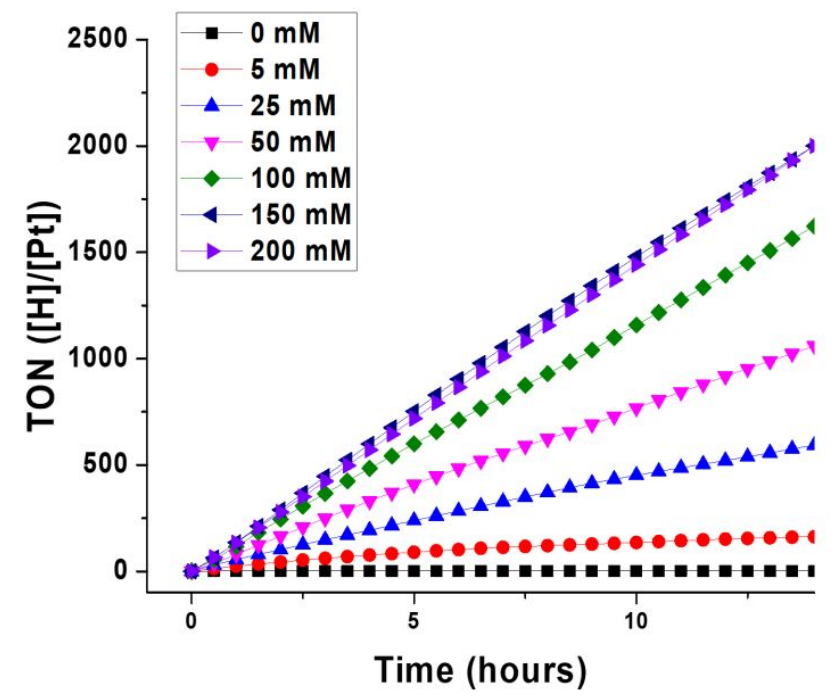

(c)

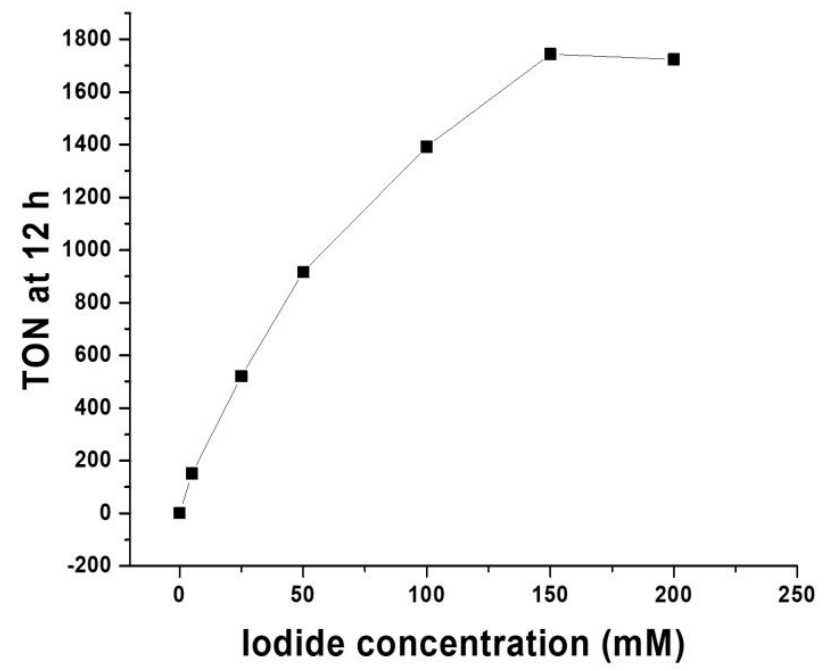

Figure S3. Hydrogen evolution curves of photocatalytic systems based on SM1 (a) with various sodium halides salts (b) with various concentration of Nal (c) TON at 12 hour after irradiation vs. iodide concentration

Hydrogen evolution curves of SM1 with $0.5 \mu \mathrm{mol}$ of $\mathrm{K}_{2} \mathrm{PtCl}_{4}$ in $13 \mathrm{~mL}$ of aqueous solution 0.92 $\mathrm{M}$ of L-ascorbic acid and no salt, $0.15 \mathrm{M}$ of NaCl, $\mathrm{NaBr}$ or various concentration of $\mathrm{Nal}$. 


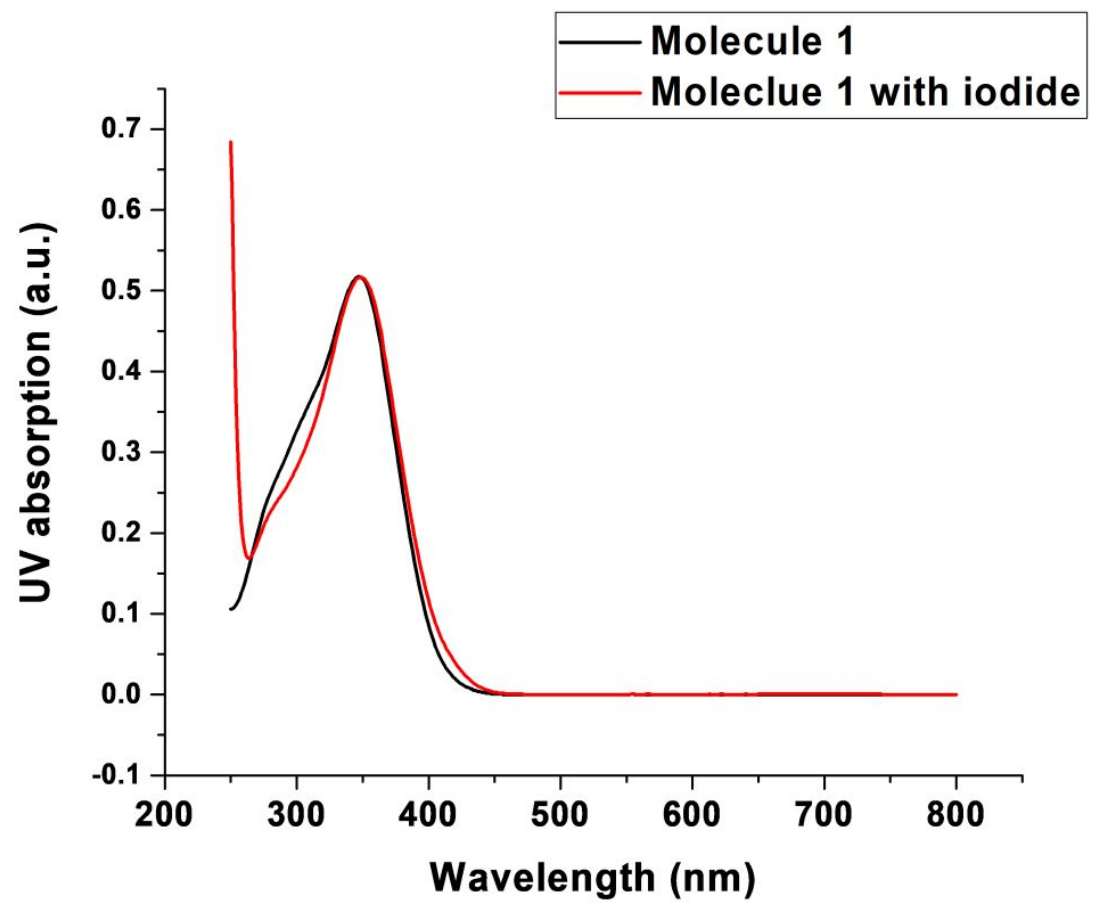

Figure S4. UV-Vis absorption spectrum of SM1 with/without iodide

UV-Vis absorption curves of $10 \mu \mathrm{M} \mathrm{SM1}$ aqueous solution and the sample with $30 \mathrm{mM}$ of $\mathrm{Nal}$.
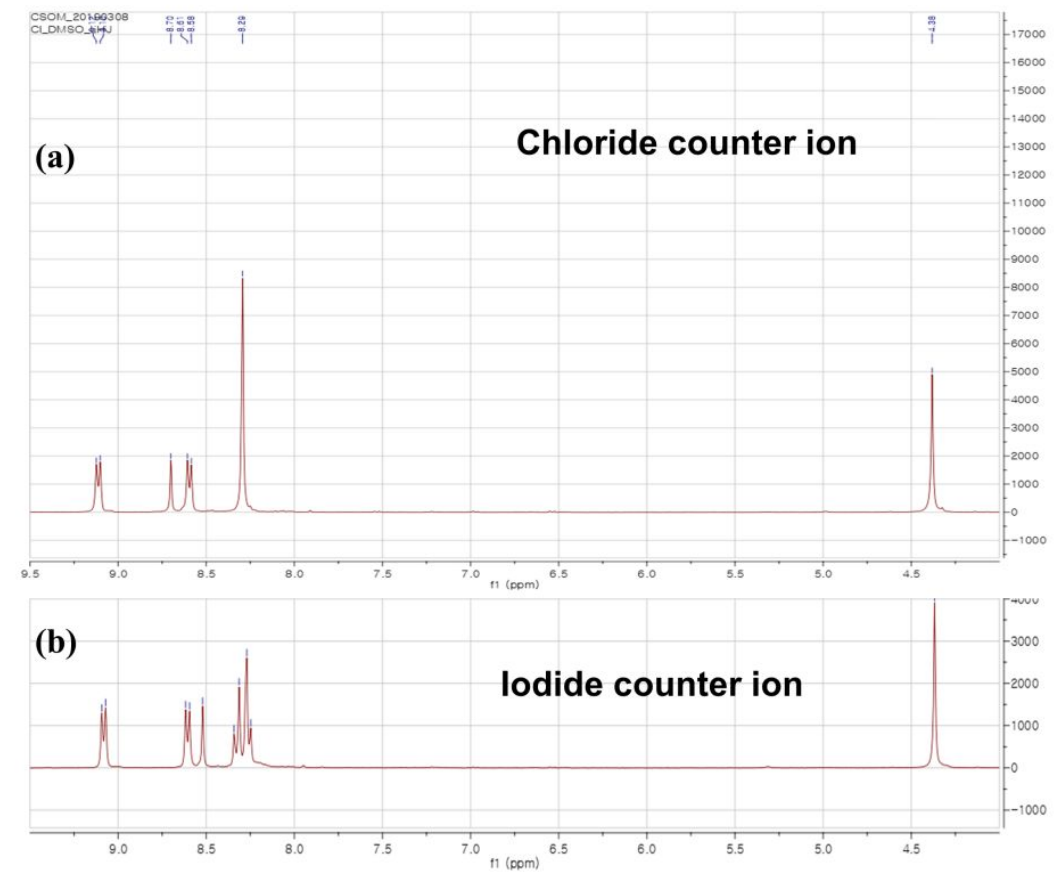

Figure S5. NMR spectrum of molecule 1 (a) with chloride counter ion and (b) with iodide counter ion in DMSO 


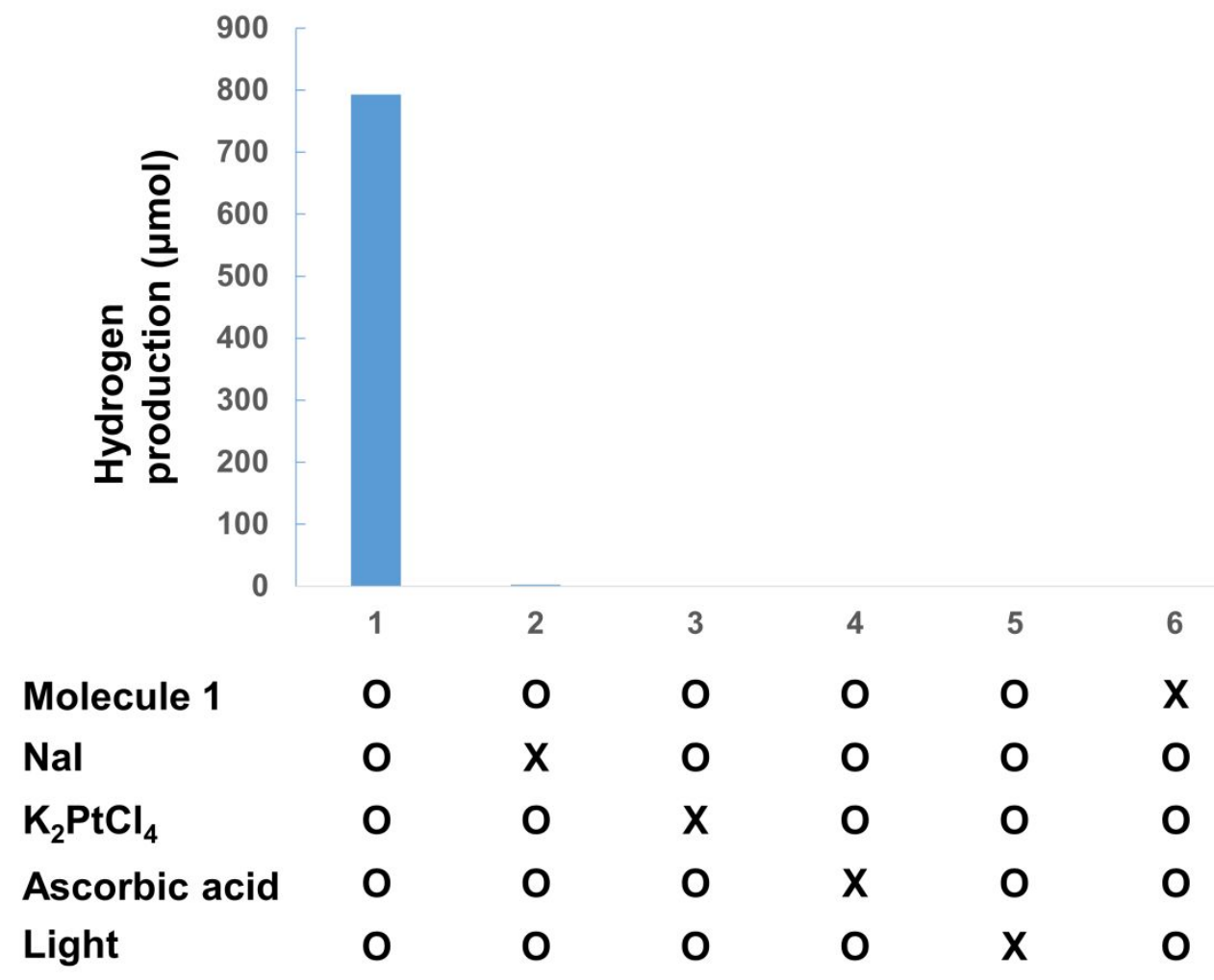

Figure S6. Hydrogen production during 24 hours in various conditions. 
(a)

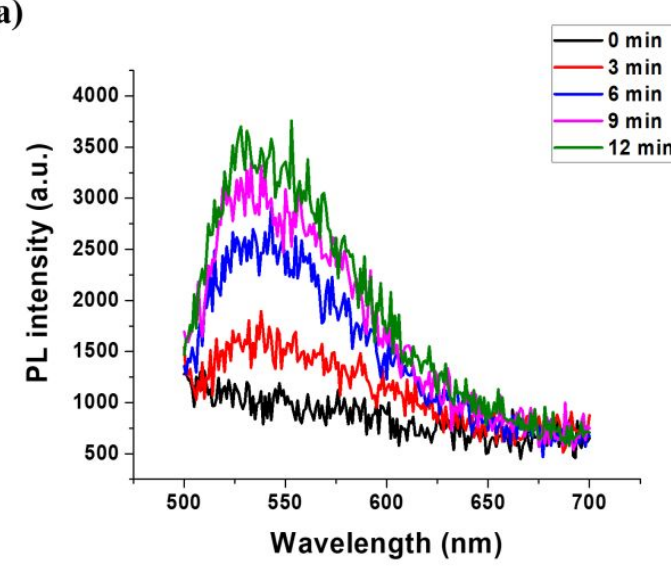

(c)

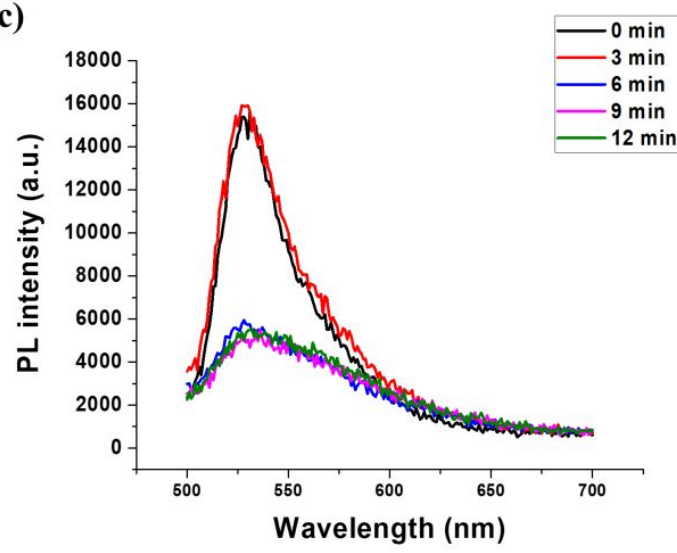

(e)

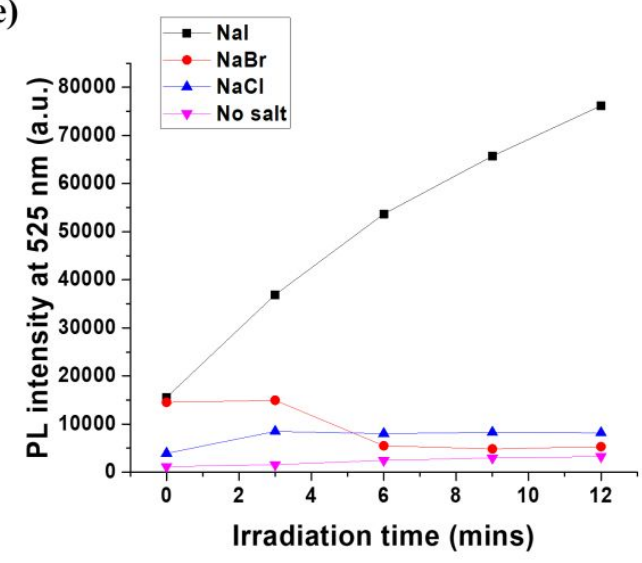

(b)

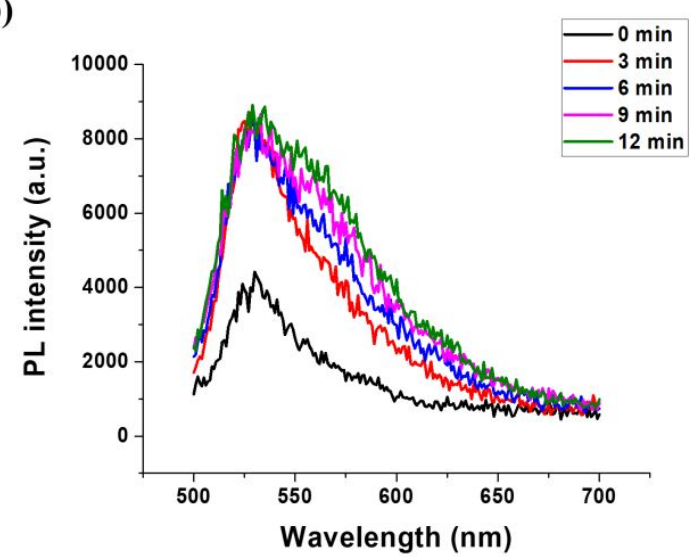

(d)

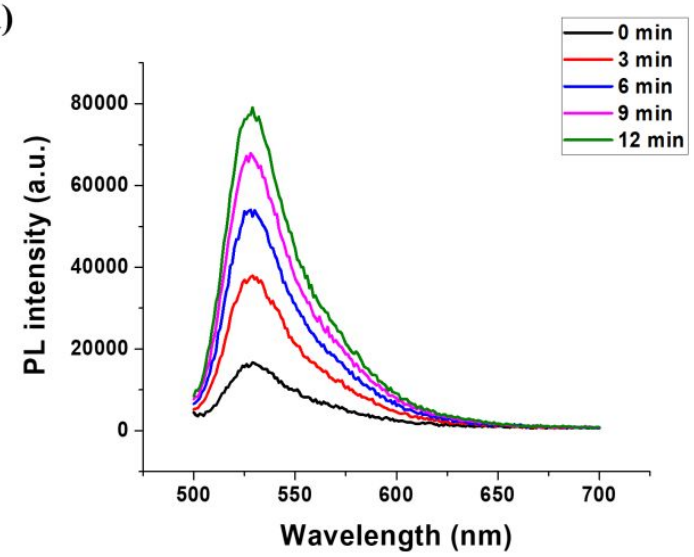

Figure S7. Singlet oxygen sensor green (SOSG) experments.

PL spectrum change upon $365 \mathrm{~nm}$ hand-held UV lamp irradiation of 0.15 micromoles 1 and 0.01 micromoles SOSG in (a) $3 \mathrm{~mL}$ aqueous solution (b) $3 \mathrm{~mL} 0.15 \mathrm{M} \mathrm{NaCl}$ aqueous solution (c) $3 \mathrm{~mL} 0.15 \mathrm{M} \mathrm{NaBr}$ aqueous solution (d) $3 \mathrm{~mL} 0.15 \mathrm{M} \mathrm{Nal}$ aqueous solution (e) PL intensity at $525 \mathrm{~nm}$ change by $365 \mathrm{~nm}$ hand-held UV lamp irradiation time 


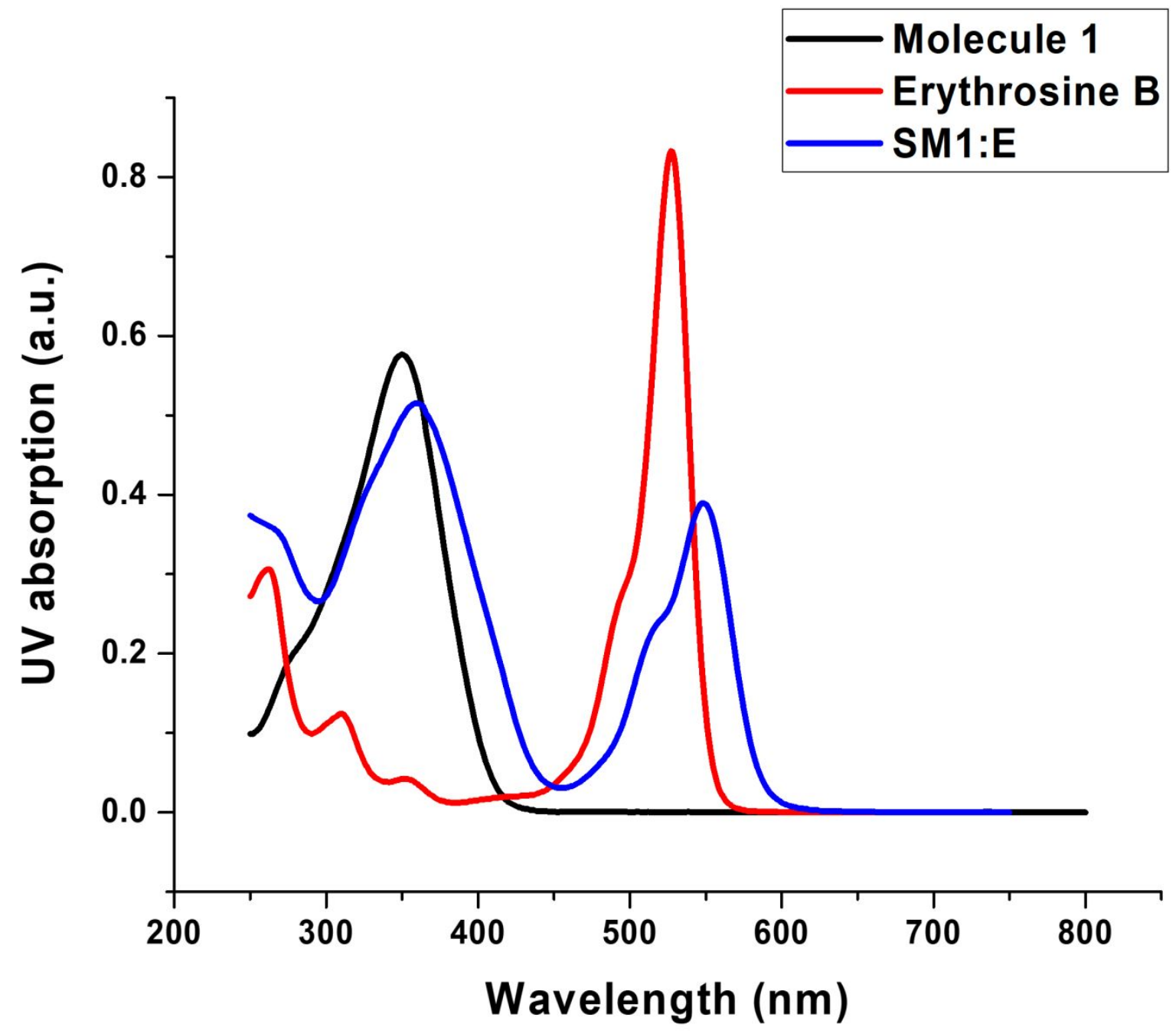

Figure S8. UV-Vis absorption spectrum of molecule 1, erythrosine B and the mixture (SM1:E).

Concentration of each molecule in each aqueous solution was $0.01 \mathrm{mM}$.

(a)

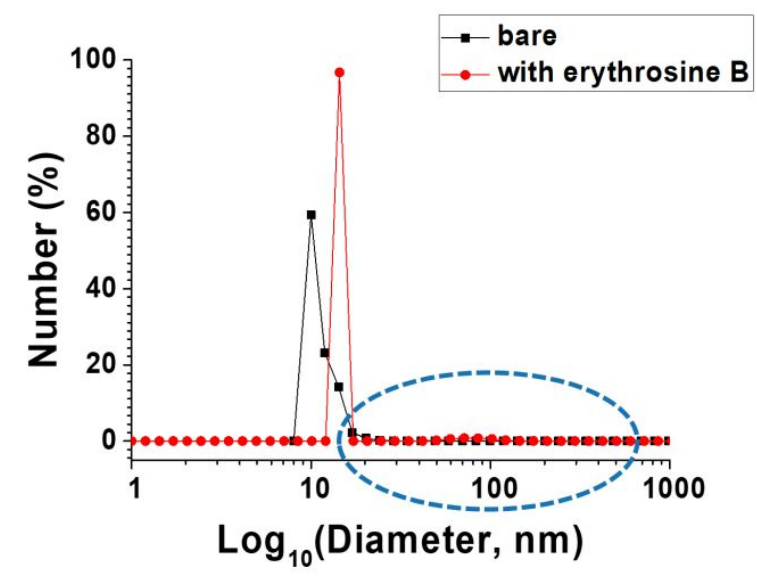

(b)

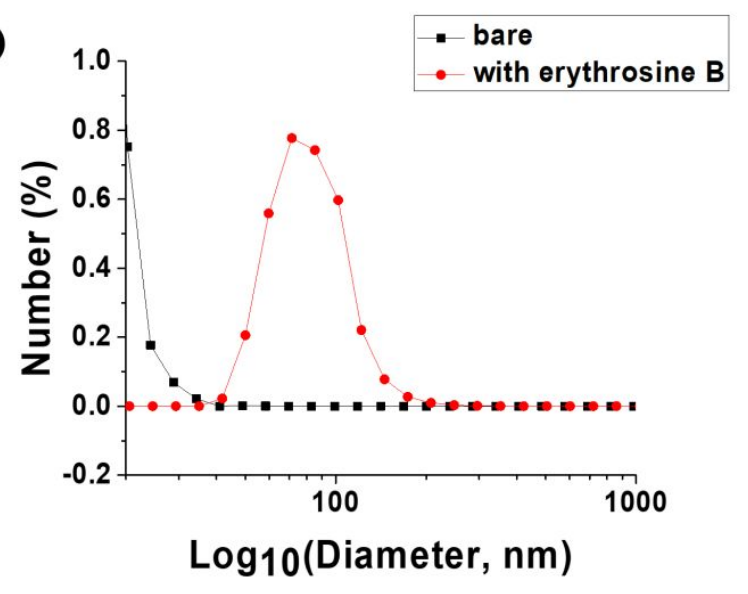

Figure S9. (a) Dynamic Light Scattering spectrum of molecule 1 (black) and mixture with erythrosine $B$ (red). (b) enlarged spectrum of marked area in (a).

Concentration of each molecule in each aqueous solution was $0.01 \mathrm{mM}$. 
(a)

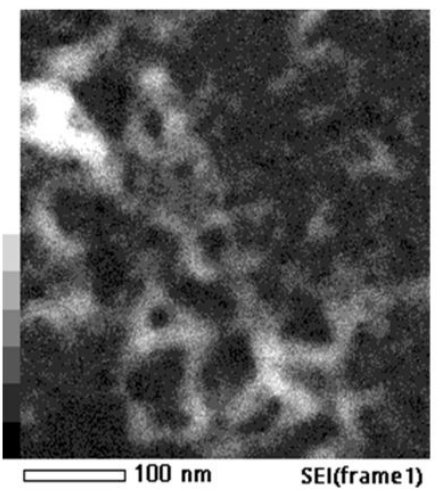

(d)

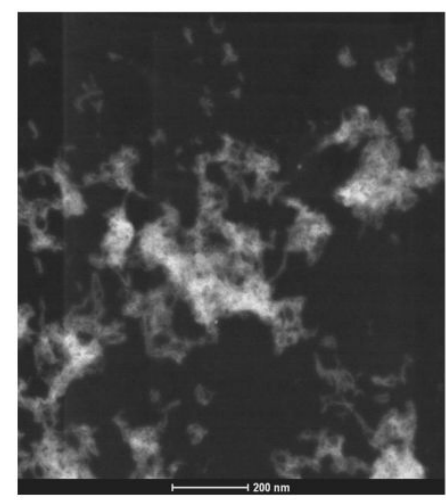

(f)

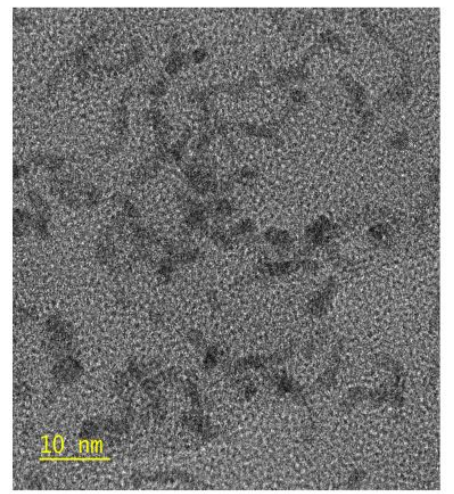

(b)

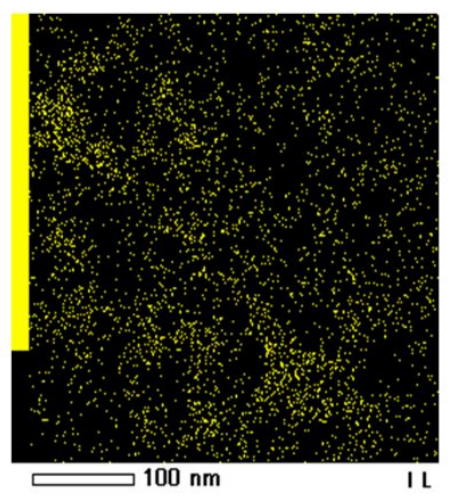

(c)

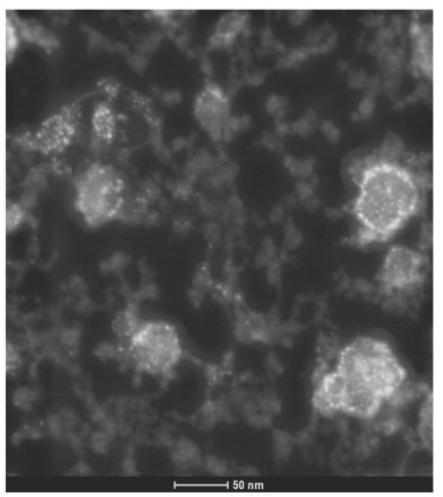

(e)
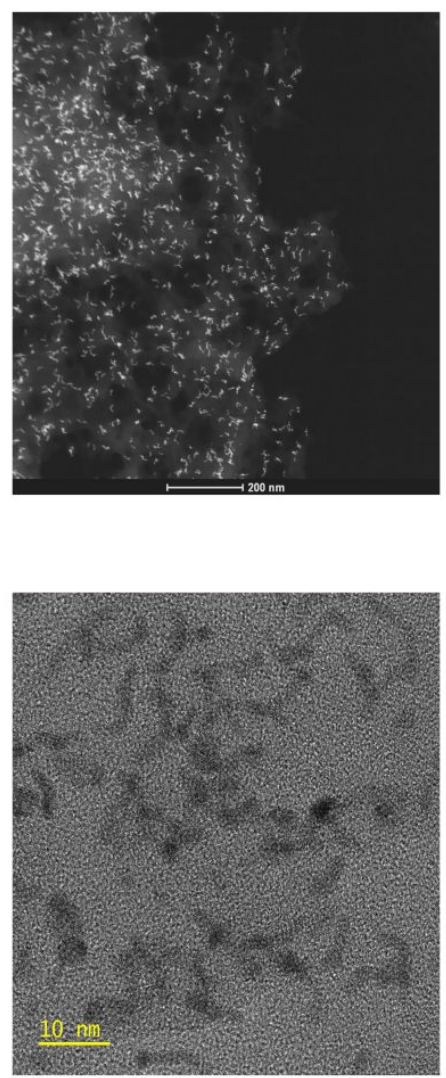
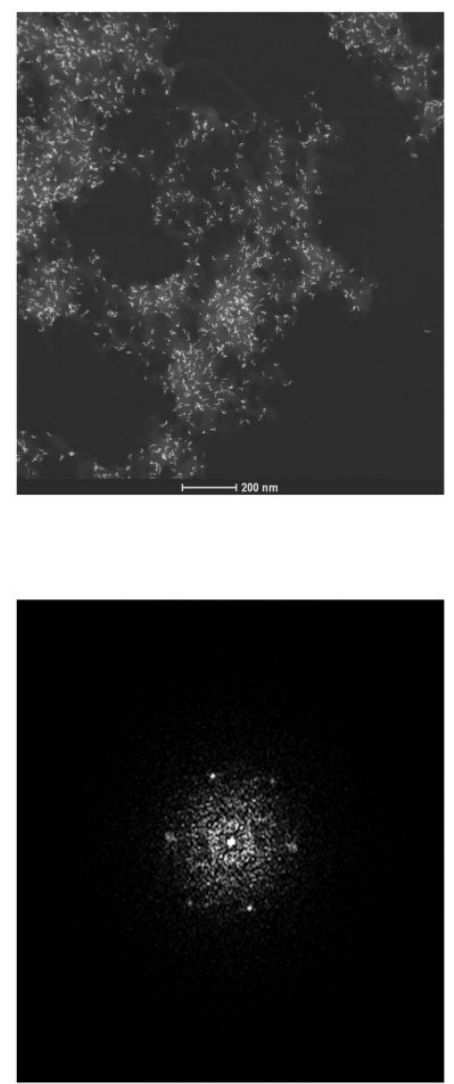

Figure S10. STEM images of dried (a) SM1:E (prepared from $50 \mu \mathrm{M}$ aqueous solution of 1 and erythrosine B) (b) EDS mapping of iodide in (a) (c) SM1:E with iodide (SM1:E in 0.15 M of $\mathrm{Nal}$ aqueous solution) (d) as prepared photocatalytic experimental sample (prepared from 0.5 $\mu \mathrm{mol}$ of $\mathrm{K}_{2} \mathrm{PtCl}_{4}, 0.65 \mu \mathrm{mol}$ of 1 and erythrosine $\mathrm{B}$, and $12 \mathrm{mmol}$ of L-ascorbic acid in $13 \mathrm{~mL}$ of $0.15 \mathrm{M}$ of $\mathrm{Nal}$ ) (e) after irradiated 24 hours (f) HRTEM images of dried SM1:E and FFT pattern of a black spot in HRTEM image. 
LUMO

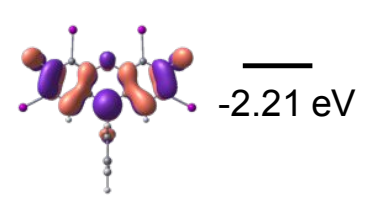



HOMO
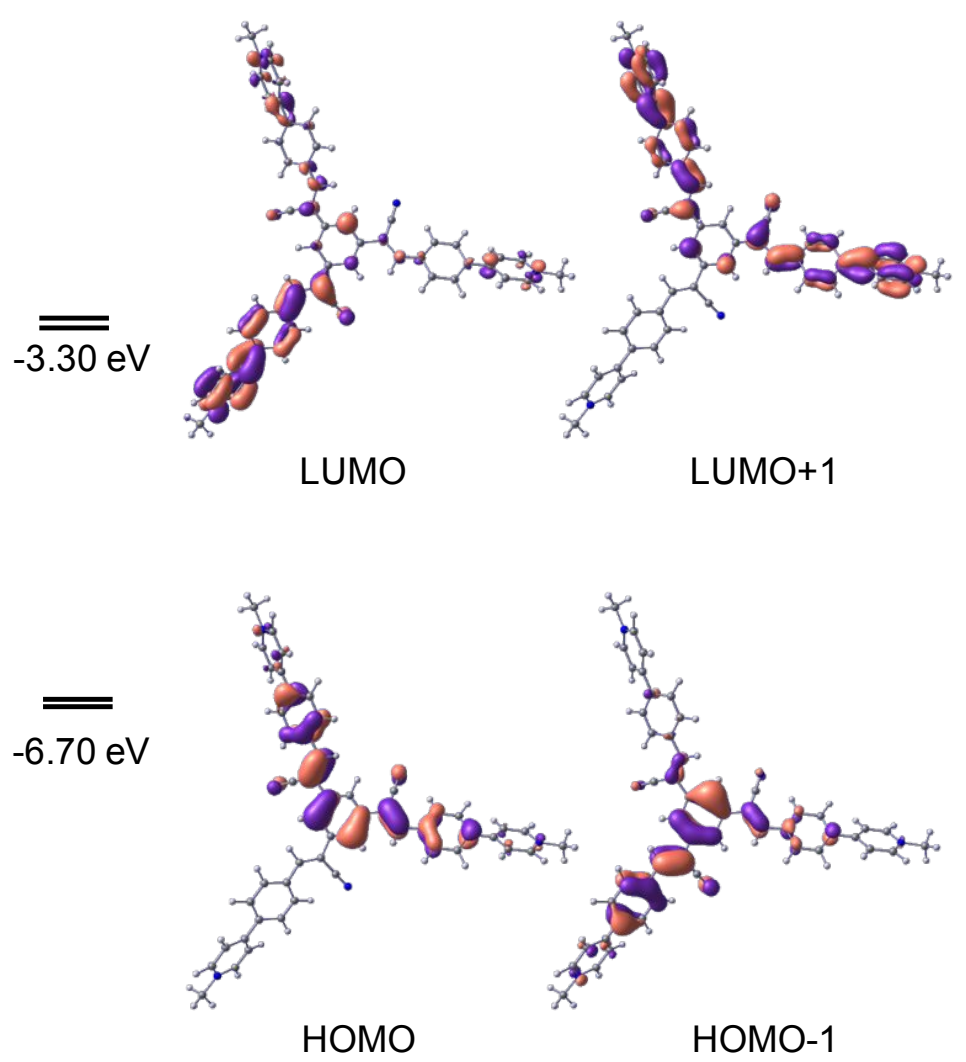

Figure S11. Frontier MO energies and topologies of E (left) and 1 (right) in water. 
(a)

E

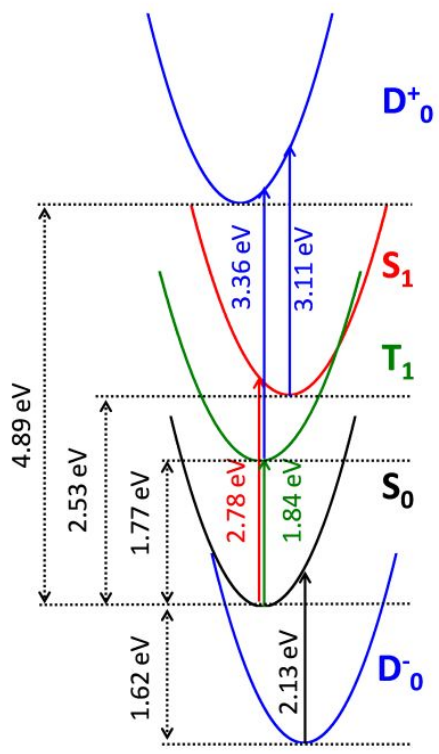

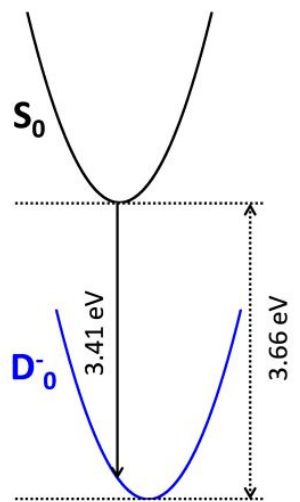

(b)

Erythrosin B

Molecule 1

SM1/I

1. Excitation:

2. ISC:

3. Reduction:

SM1:E

1. Excitation:

2. Charge Sep.:

3. Charge Rec.:$$
\text { e driving force }
$$

$\mathrm{D}_{0}^{+}(\mathbf{E}) \rightarrow \mathrm{S}_{0}(\mathbf{E})$

$\mathrm{D}_{0}^{-}(\mathbf{1}) \rightarrow \mathrm{S}_{0}(\mathbf{1})$

$120 \mathrm{ps}$

fast against charge migration to $P t$

SM1:E/I

1. Excitation:

2.ISC:

$\mathrm{S}_{0}(\mathbf{E}) \rightarrow \mathrm{S}_{1}(\mathbf{E}) 2.53 \mathrm{eV}\left(\mathrm{E}_{00}\right)$

$\mathrm{S}_{1}(\mathbf{E}) \rightarrow \mathrm{T}_{1}(\mathbf{E})-0.76 \mathrm{eV}\left(\mathrm{E}_{00}\right)$

$\mathrm{T}_{1}(\mathbf{E}) \rightarrow \mathrm{D}_{0}{ }^{+}(\mathbf{E}) 3.12 \mathrm{eV}\left(\mathrm{E}_{00}\right)$

$3.36 \mathrm{eV}\left(\mathrm{E}_{\mathrm{vert}}\right)$

small driving force

3. Red. by SE:

4. Redox:

$\mathrm{T}_{1}(\mathbf{E}) \rightarrow \mathrm{D}_{0}-(\mathbf{E})-3.39 \mathrm{eV}\left(\mathrm{E}_{00}\right)$

$\mathrm{D}_{0}^{-}(\mathbf{E}) \rightarrow \mathrm{S}_{0}(\mathbf{E}) 1.62 \mathrm{eV}\left(\mathrm{E}_{00}\right)$

$$
2.13 \mathrm{eV}\left(\mathrm{E}_{\mathrm{vert}}\right)
$$

5. Ox. $\left(\mathrm{H}_{2}\right.$ evolu. $)$ :

\section{$<$ fs}

$<170$ fs

$\mathrm{S}_{0}(\mathbf{1}) \rightarrow \mathrm{D}_{0}^{-}(\mathbf{1})-3.66 \mathrm{eV}\left(\mathrm{E}_{00}\right)$ (slow)

$-3.41 \mathrm{eV}\left(\mathrm{E}_{\mathrm{vert}}\right)$

slow

$\mathrm{S}_{0}(\mathbf{1}) \rightarrow \mathrm{D}_{0}^{-}(\mathbf{1})-3.66 \mathrm{eV}\left(\mathrm{E}_{00}\right)$

$-3.41 \mathrm{eV}\left(\mathrm{E}_{\mathrm{vert}}\right)$

$\mathrm{D}_{0}^{-}(\mathbf{1}) \rightarrow \mathrm{S}_{0}(\mathbf{1}) \quad 3.66 \mathrm{eV}\left(\mathrm{E}_{00}\right)$ large driving force

large driving force

Figure S12. (a)Adiabatic and vertical transition energies for states of interest in E (left) and 1 (right). (b) Equations for all steps of the photocatalytic processes.

$\mathrm{S}_{0}=$ singlet ground state, $\mathrm{S}_{1}=$ first excited singlet state, $\mathrm{T}_{1}=$ first excited triplet state, $\mathrm{D}^{+}{ }_{0}=$ radical cation, $\mathrm{D}_{0}^{-}=$radical anion. 

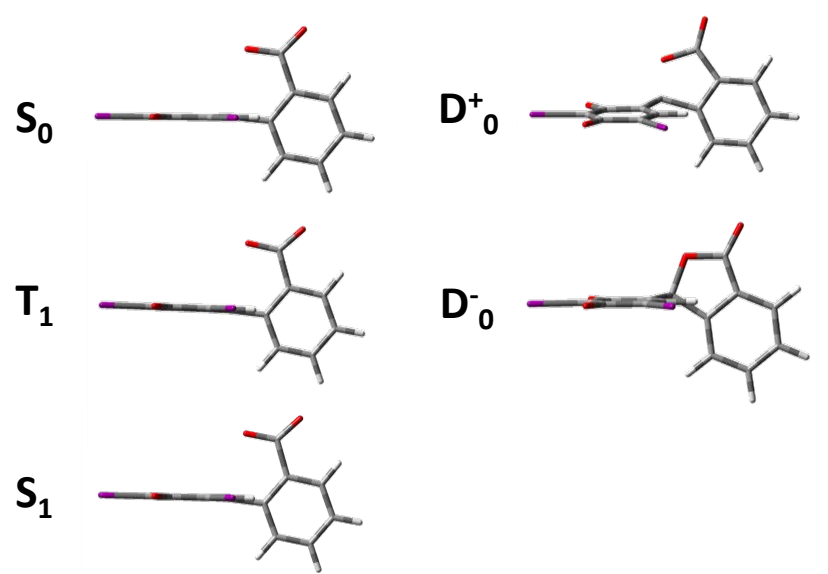

Figure S13. Results of the geometry optimizations (in water) for $E$ in the different neutral and charged electronic states.
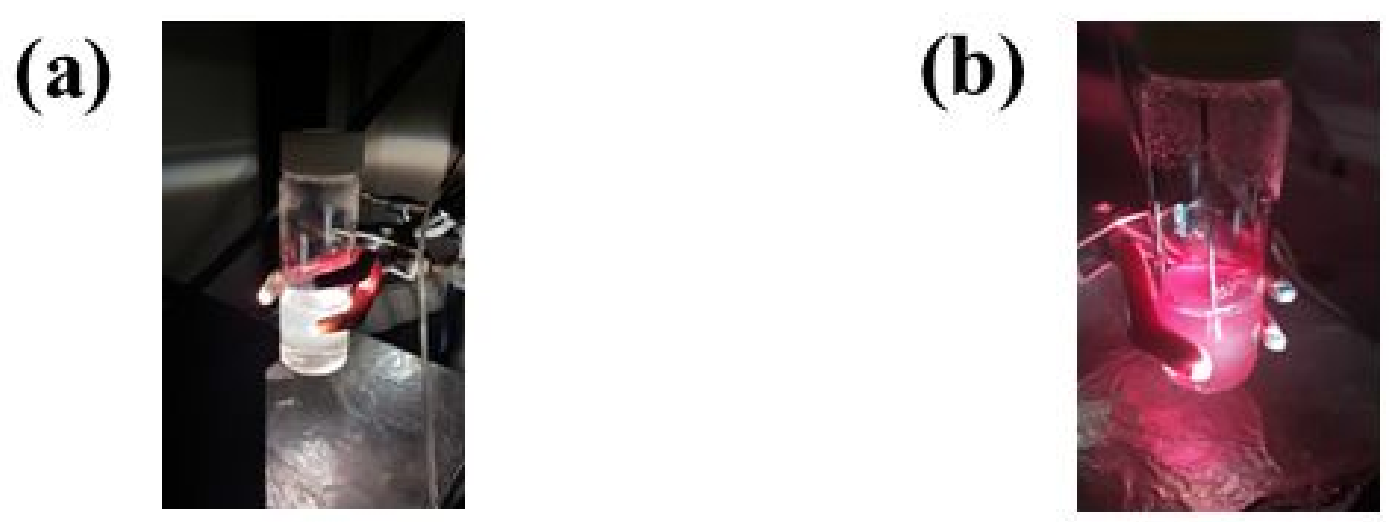

Figure S14. Pictures of photocatalytic systems under visible light irradiation.

(a) Sample without molecule 1 (prepared from $0.5 \mu \mathrm{mol}$ of $\mathrm{K}_{2} \mathrm{PtCl}_{4}, 0.65 \mu \mathrm{mol}$ of Erythrosine B and $12 \mathrm{mmol}$ of L-ascorbic acid with $0.15 \mathrm{M}$ of Nal in $13 \mathrm{~mL}$ of distilled water) after 10 minutes of visible light irradiation. Because erythrosine radical anion is very unstable upon visible light irradiation, the sample was turned to be white. (b) Sample with molecule 1, SM1:E/l (prepared from $0.5 \mu \mathrm{mol}$ of $\mathrm{K}_{2} \mathrm{PtCl}_{4}, 0.65 \mu \mathrm{mol}$ of Erythrosine B and Molecule 1, $12 \mathrm{mmol}$ of L-ascorbic acid with $0.15 \mathrm{M}$ of $\mathrm{Nal}$ in $13 \mathrm{~mL}$ of distilled water) after 10 hours of visible light irradiation. 


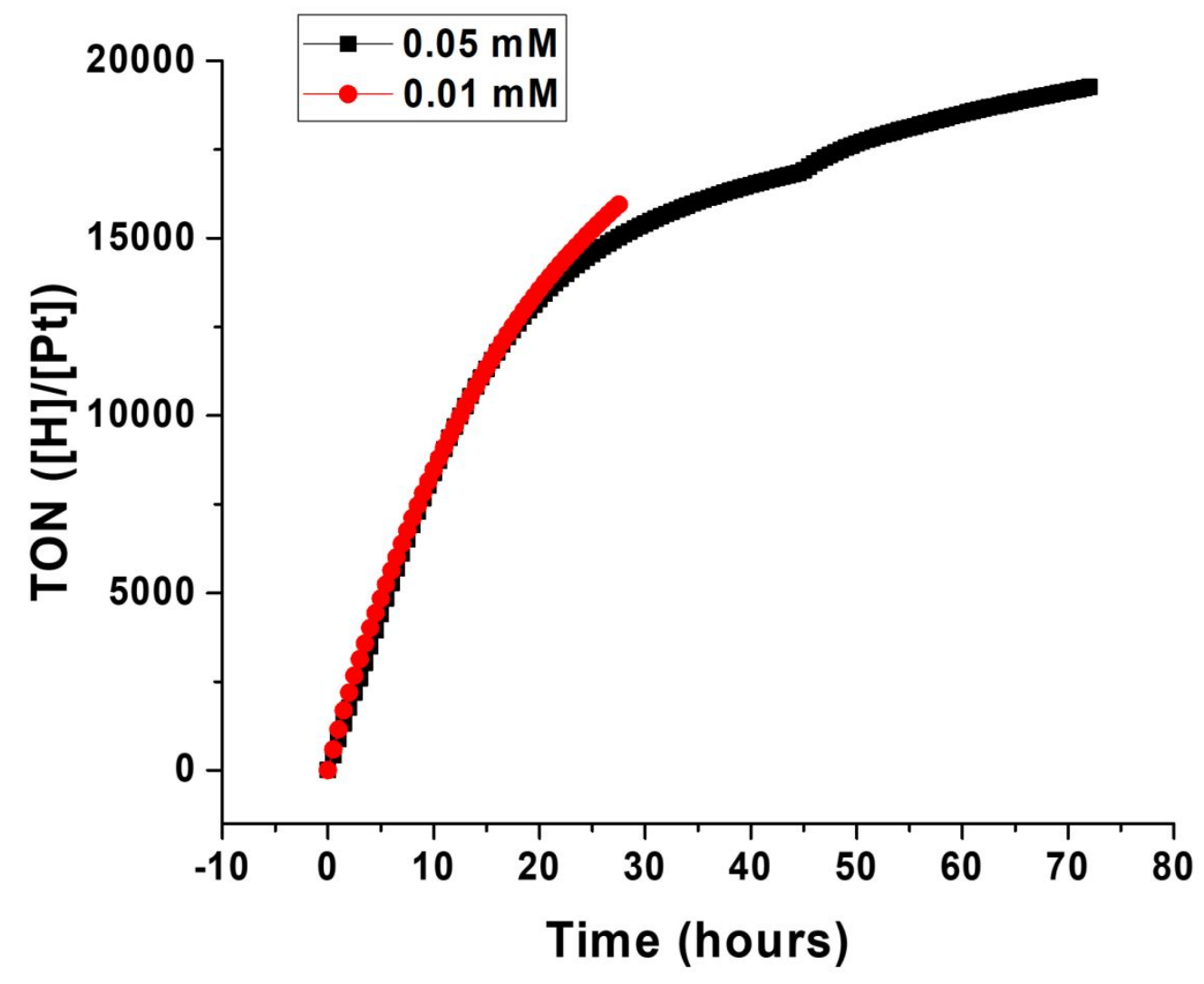

Figure S15. Hydrogen evolution curves of SM1:E/I with various concentration.

$0.01 \mathrm{mM}$ of molecule 1 (red) and $0.05 \mathrm{mM}$ of molecule 1 (black) (Other additives were also changed in proportion). 
(a)

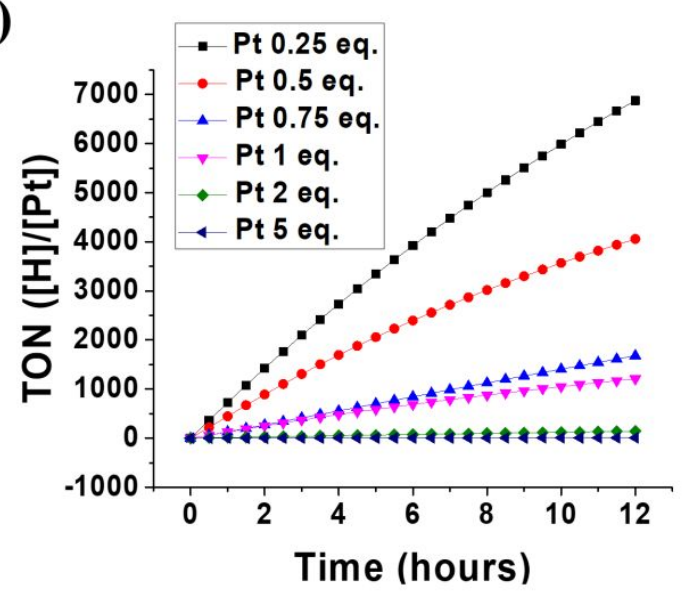

(b)

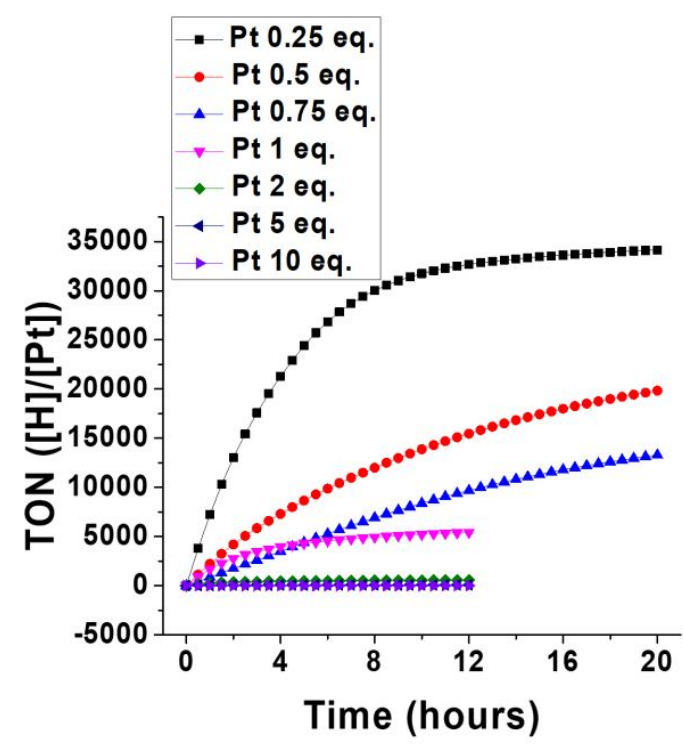

Figure S16. Turnover number of (a) SM1/l and (b) SM1:E/l with various amounts of platinum.

Each legend shows the equivalent of platinum to molecule 1.

(a)

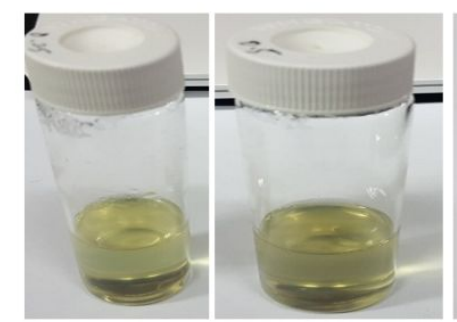

Pt: 0.25 eq. 0.5 eq.

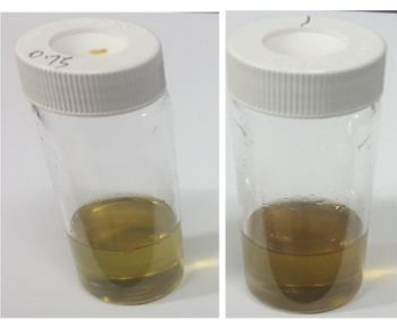

0.75 eq. 1 eq.

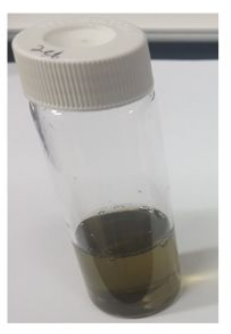

2 eq.

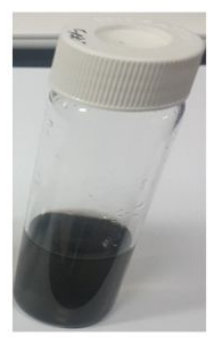

5 eq.

(b)

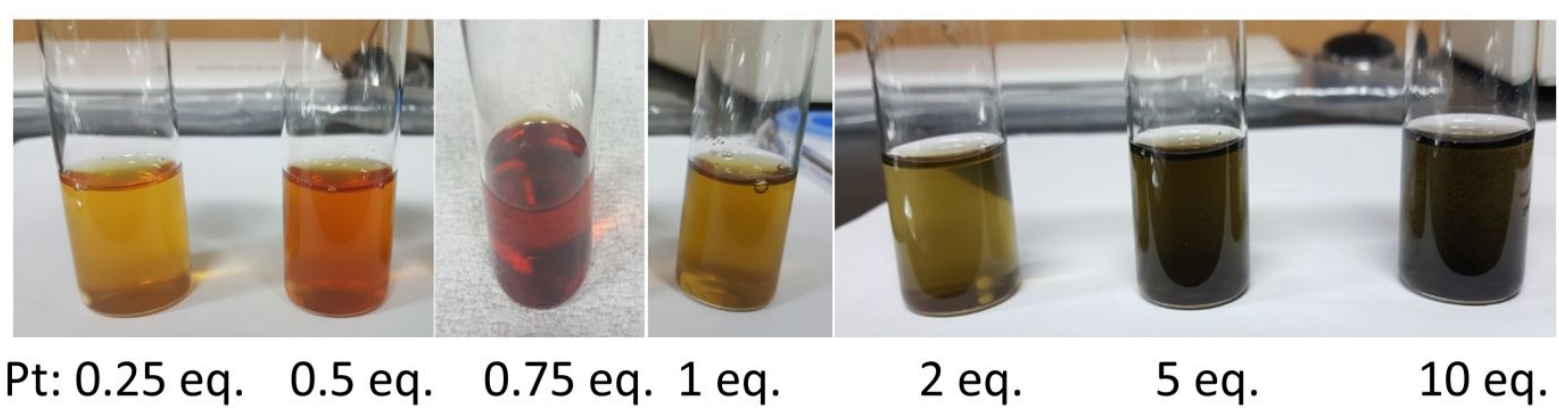

Figure S17. Pictures of (a) SM1/E and (b) SM1:E/l with various amounts of platinum after 24 hours of visible light irradiation.

The numbers under each picture show the equivalent of platinum to molecule 1 . 
(a)

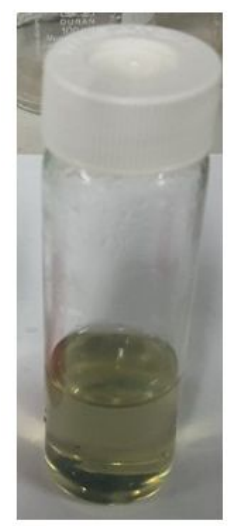

(b)

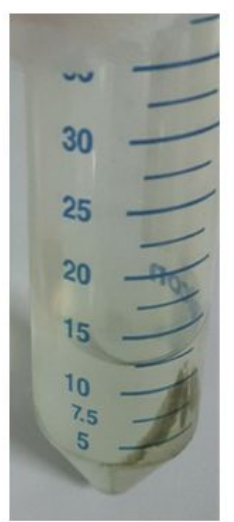

(c)

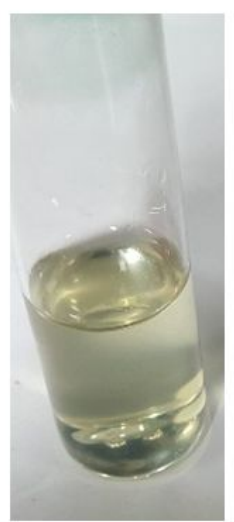

(d)

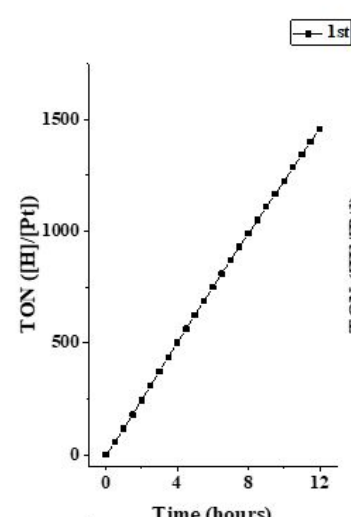

$\rightarrow-1$ st
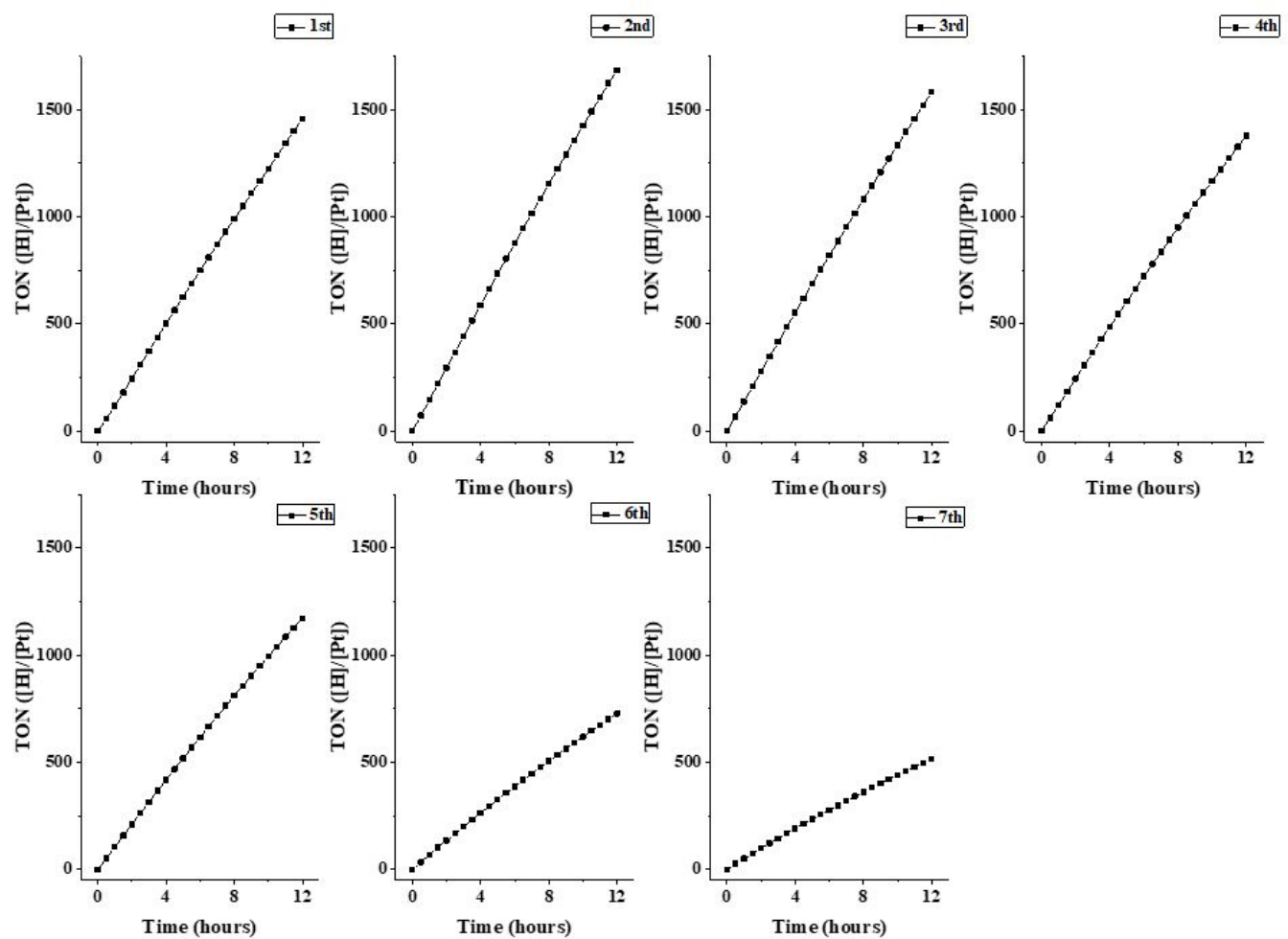

Figure S18. Pictures of the SM1/l solution and hydrogen evolution curves.

Pictures of (a) After 12 hours hydrogen evolution reaction. (b) Centrifuged (8000 rpm, 20 mins) sample in A. (c) Re-distiributed in fresh $13 \mathrm{~mL}$ of aqueous solution of $0.15 \mathrm{M} \mathrm{Nal}$ and $0.92 \mathrm{M} \mathrm{L}$ ascorbic acid (d) Hydrogen evolution curves 7 cycles (12 hour for each cycle). 
(a)

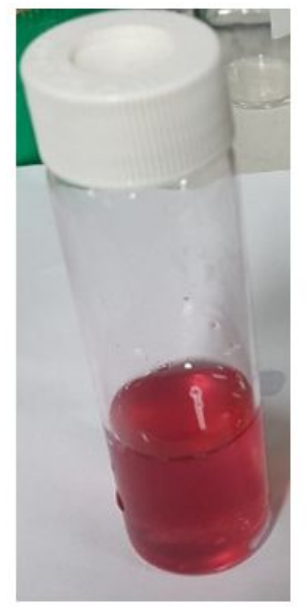

(b)

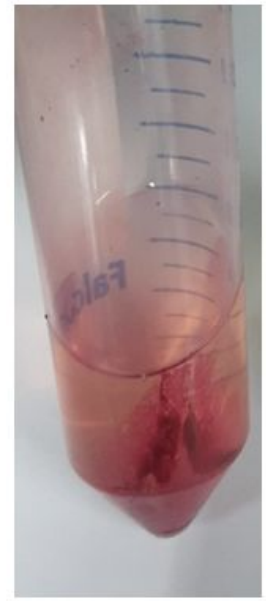

(c)
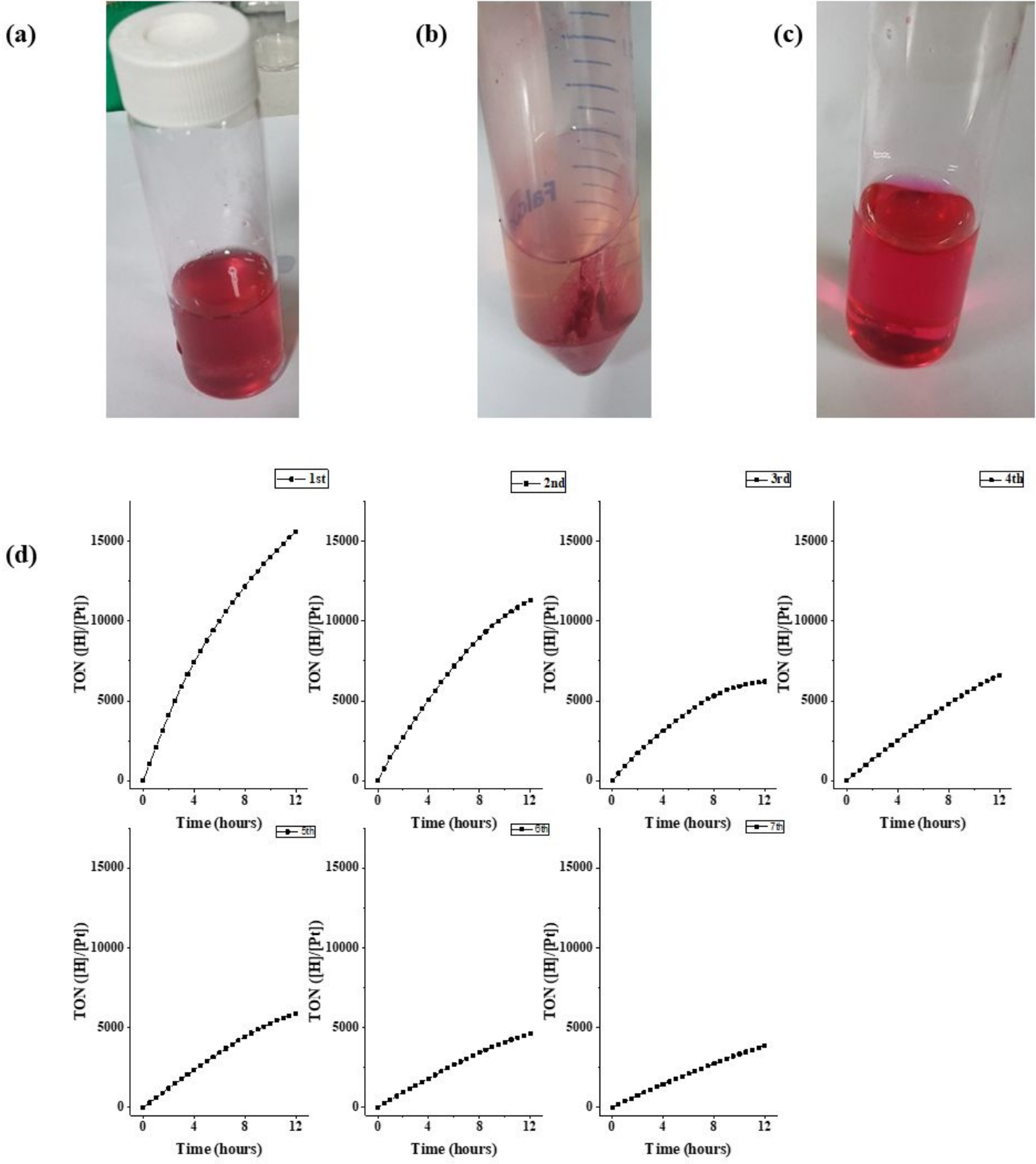

$-2 \mathrm{nd}$
$-3 \mathrm{rd}$

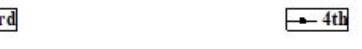

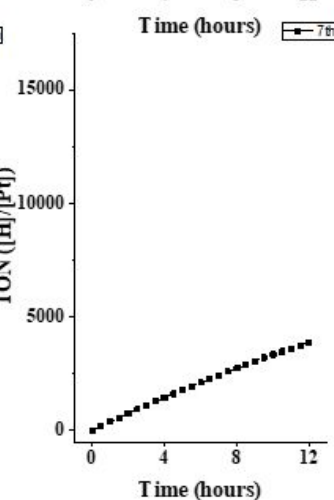

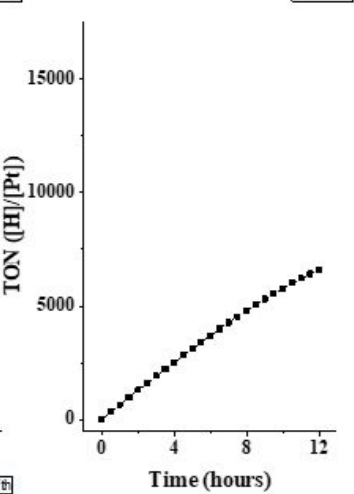

Figure S19. Pictures of the SM1:E/l solution and hydrogen evolution curves.

Pictures of (a) After 12 hours hydrogen evolution reaction. (b) Centrifuged (8000 rpm, 20 mins) sample in (a). (c) Re-distiributed sample in fresh $13 \mathrm{~mL}$ of aqueous solution of $0.15 \mathrm{M} \mathrm{Nal}$ and 0.92 M L-ascorbic acid (d) Hydrogen evolution curves 7 cycles (12 hours for each cycle). 

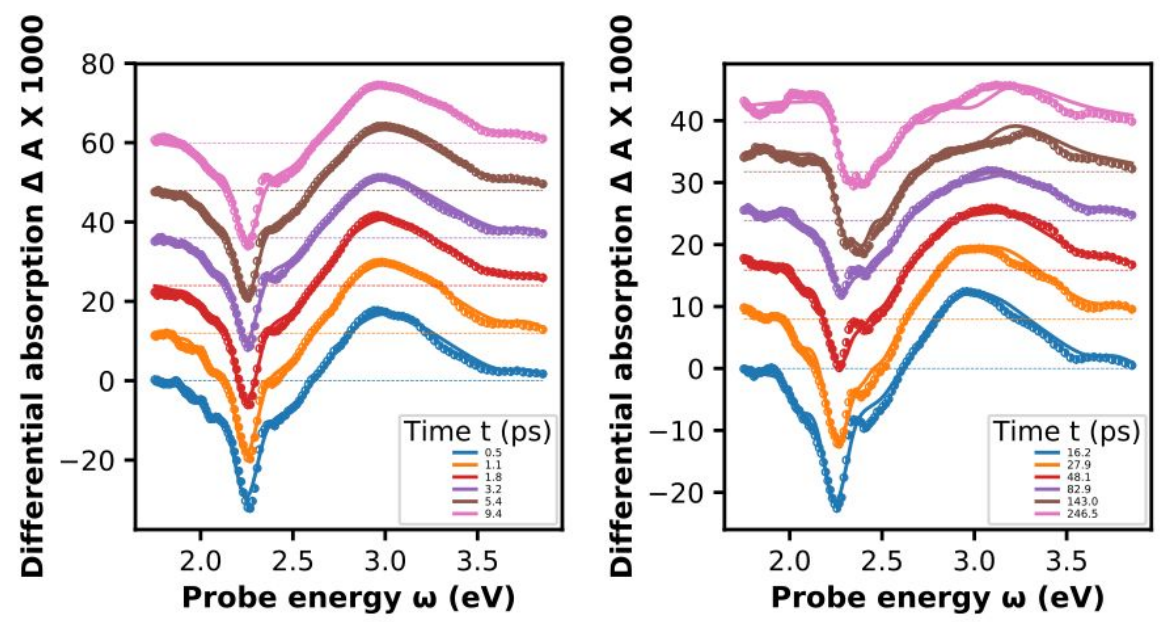

Figure S20. Transient absorption (TA) spectra of erythrosine.

Concentration: $0.05 \mathrm{mM}$ aqueous solution; pumping at $2.3 \mathrm{eV}$ with $150 \mathrm{fs}$ pulses of $450 \mathrm{~nJ}$ pulse energy, at different pump-probe delay times, as given in the inset (symbols). Lines are fits obtained by matrix-multiplying the solid lines in Figure S21 a and Figure S21 b,c, of same color, according to eq. 5 .

a) SVD cross-sections

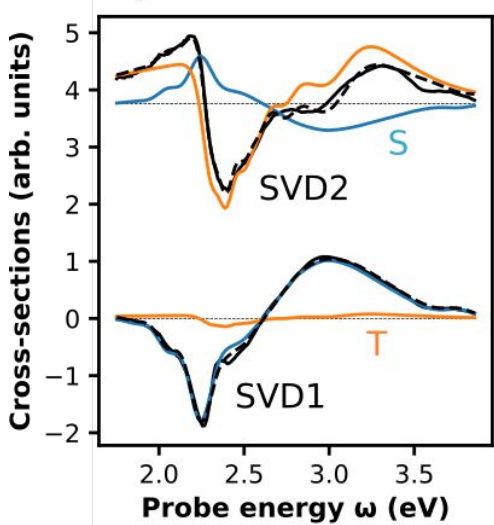

b) Singlet dynamics

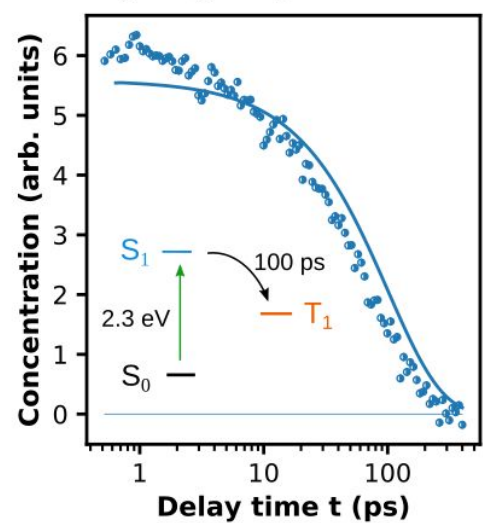

c) Triplet dynamics

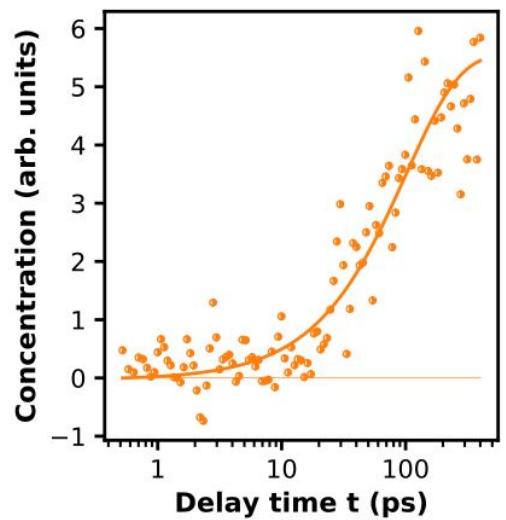

Figure S21. Spectral modeling of erythrosine. (a) Characteristic spectra of a singular value decomposition (SVD) of the time-resolved TA spectrum (symbols in Figure S20) (black solid line) and reconstruction by fitting photophysical model spectra of singlet (blue) and triplet (orange) states (black dashed lines). (b) Resulting time-resolved concentrations of singlet and (c) triplet states.

In Figure S20, we show TA spectra of E in solution. The spectral evolution from the singlet to the triplet state is well documented in the literature ${ }^{2,3}$ and serves as a benchmark for our method. For the spectral model of the lowest singlet excited state, we fit only the first 5 ps where we know that triplet production has not yet started. We obtain a good fit assuming the presence of transient photobleach (PB) and stimulated emission (SE) of approximately equal strength and a significant Stokes shift which is well documented in the literature. ${ }^{4,} 5$ Moreover, a strong photoinduced absorption with a high Huang-Rhys factor is found at $2.7 \mathrm{eV}$. For all 
transitions, we assumed a similar energetic spacing dc $=0.185-0.187 \mathrm{eV}$ of an effective vibronic progression. The parameters are summarized in table S1.

We did not find a time range in our experiment in which we could be safe that only triplet states are conserved; therefore, we fitted the whole measured range and fixed the spectral parameters of the singlet state to those obtained at short times. We found that in order to get a good fit over the whole measure time range (see Figure S21), we had to include a total of 4 PA bands for the triplet state. The PA region is dominated by a very broad band PAT2 centered at $2.41 \mathrm{eV}$ and a Gaussian width $\mathrm{b}_{\mathrm{g}}$ exceeding $1 \mathrm{eV}$. Furthermore, we note that the width of the PB of the triplet state is much smaller than that of the singlet state. In solution, where static hole burning effects and energy transfer in a disordered density of states cannot take place, the spectral characteristics of PB should be the same for all excited states of a molecule. However, a look at Figure S20 confirms that the vibronic replica PB (01) at $2.6 \mathrm{eV}$ becomes sharper at later times; justifying this fitting result.

Table S1. Spectral model parameters for the singlet and triplet states in Figure S21. The significance of the fitting parameters is as follows. a: spectral weight; bg: Gaussian width; c: center energy; dc: energetic spacing of an effective progression (note: negative for stimulated emission); hr: Huang-Rhys factor. Parameters in red deserve attention and are discussed in the text.

\begin{tabular}{|c|c|c|c|c|c|c|}
\hline \multicolumn{7}{|c|}{ State: S in phase: erythrosine, soln } \\
\hline PAS1 & a: 2.727 & bg: 0.287 & c: 2.703 & dc: 0.187 & hr: 1.611 & s: 0.605 \\
\hline PAS2 & a: 0.079 & bg: 0.150 & c: 3.766 & dc: 0.187 & hr: 0.000 & s: 0.000 \\
\hline PB & a: -1.000 & bg: 0.353 & c: 2.340 & dc: 0.187 & hr: 0.500 & s: 0.000 \\
\hline SE & a: -1.204 & bg: 0.125 & c: 2.241 & dc: -0.187 & hr: 0.331 & s: 0.000 \\
\hline \multicolumn{7}{|c|}{ State: T in phase: erythrosine, sol } \\
\hline PB & a: -1.000 & bg: 0.133 & c: 2.353 & dc: 0.187 & hr: 0.600 & s: 0.000 \\
\hline PAT1 & a: 0.053 & bg: 0.076 & c: 2.554 & dc: 0.185 & hr: 0.003 & s: 0.240 \\
\hline PAT2 & a: 1.049 & bg: 1.189 & c: 2.411 & dc: 0.185 & hr: 0.010 & s: $: 0.680$ \\
\hline PAT3 & a: 0.413 & bg: 0.407 & c: 3.275 & dc: 0.185 & hr: 0.000 & s: 1.093 \\
\hline PAT4 & a: 0.104 & bg: 0.074 & c: 2.348 & dc: 0.000 & hr: 0.000 & s: 0.000 \\
\hline
\end{tabular}



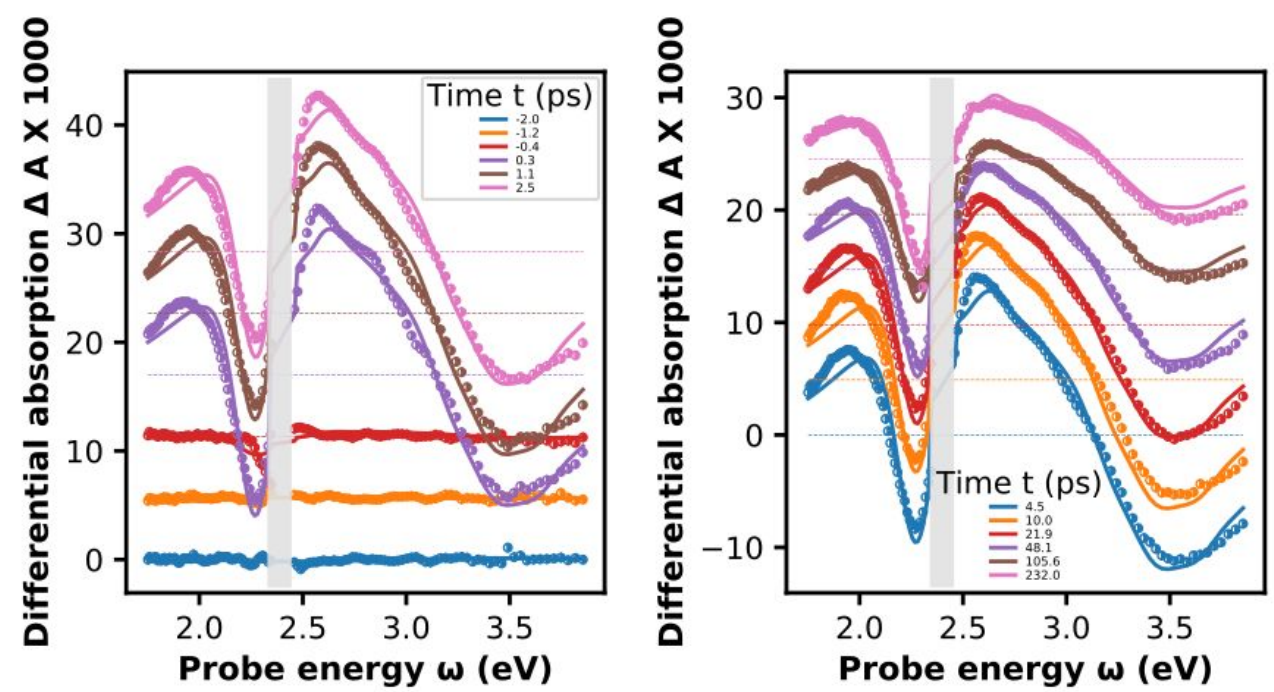

Figure S22. Transient absorption (TA) spectra of SM1:E. Pump energy $2.3 \mathrm{eV}$ with $150 \mathrm{fs}$ pulses of $450 \mathrm{~nJ}$ pulse energy, at different pump-probe delay times, as given in the inset (symbols). Lines are fits obtained by matrix-multiplying the solid lines in Figure S23 a and Figure $\mathrm{S} 23 \mathrm{~b}, \mathrm{c}$, of same color, according to eq. 5 . Grey bar indicates region of pump pulse scattering.

a) SVD cross-sections

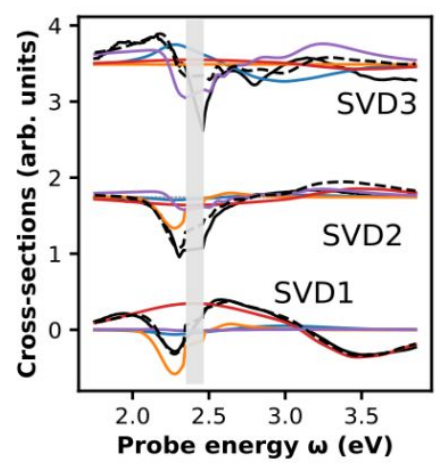

b) Singlet dynamics

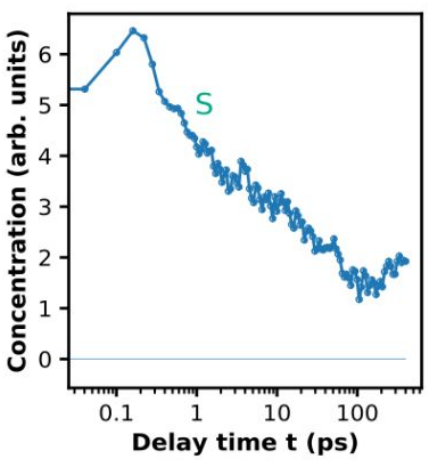

c) Charge and triplet dynamics

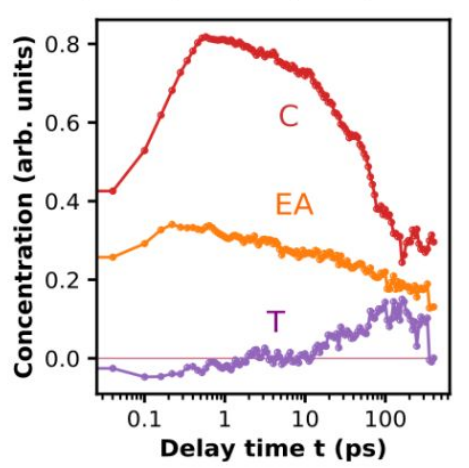

Figure S23. Spectral decomposition of SM1:E. (a) Characteristic spectra of a singular value decomposition (SVD) of the time-resolved TA spectrum (symbols in Figure S22) (black solid line) and reconstruction by fitting photophysical model spectra of singlet (blue), electroabsorption (EA) of erythrosin (orange), charges in molecule 1 (red) and triplet (green) states (black dashed lines). (b) and (c) Resulting time-resolved concentrations. Grey bar indicates region of pump pulse scattering.

In Figure S22, we show TA spectra in SM1:E without iodide, after pumping at $2.3 \mathrm{eV}$ with 150 $\mathrm{fs}, 450 \mathrm{~nJ}$ pulses. The most striking feature, as discussed in the main text, is the immediate presence of PB of the SM1 although the pump pulse is in resonance only with erythrosine B. 
Since ultrafast excitation energy transfer is excluded based on energetic reasoning, we assign this phenomenon to ultrafast charge transfer from E to SM1, creating a charge separated state. We manage to fully reproduce the TA spectra at all times (see Figure S22) by including the first derivative of the PB of Erythrosine B; this electroabsorption EA is well-known for organic donor-acceptor compounds ${ }^{[4]}$ in which the static electric field causes a dynamic Stark shift for neutral molecules in the vicinity. In Table S2, we give the parameters for the contributing photoexcited states. Note that the charge on $E$ is defined by a combination of EA (first derivative) and PB ( $0^{\text {th }}$ derivative) which is strictly only correct if the cation-anion separation does not change, as otherwise the relation between EA and charge density (PB) is no longer proportional. ${ }^{5}$ Note also that we took the spectral parameters of the singlet and triplet states of $\mathbf{E}$ from table $\mathbf{S 1}$, allowing some small changes in the parameters due to the transition into the solid state. In Figure S24, we fitted the charge decay and the build-up of triplet states with a simple first-order kinetics, which suggests that the triplets are produced by charge recombination, albeit at low yields (approx. 20\%)

a) Charge dynamics

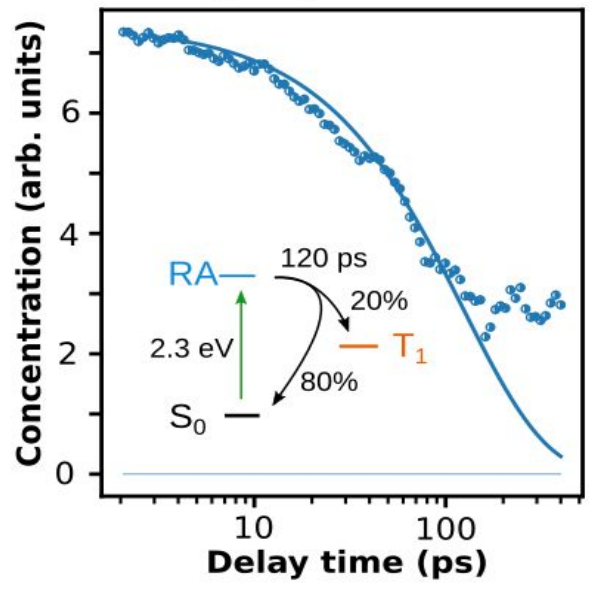

b) Triplet dynamics

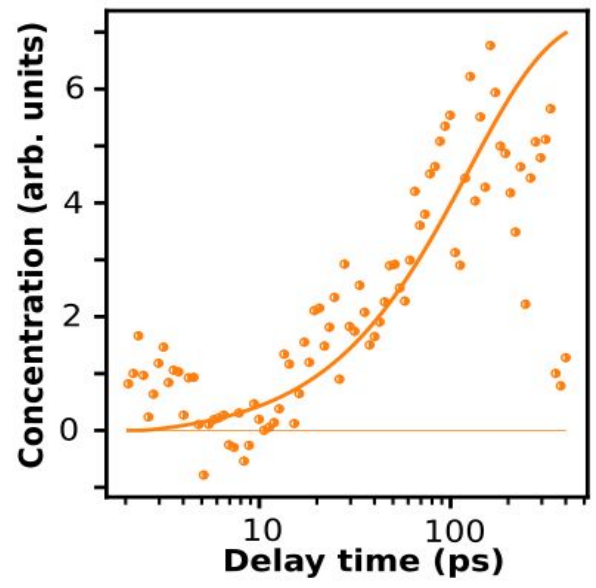

Figure S24. Kinetic modeling using only the charge and triplet dynamics from Figure $S 23$ (red and violet curves, respectively) from $t=2$ ps onward. 
Table S2. Spectral model parameters for the states in Figure S23. The significance of the fitting parameters is as follows. a: spectral weight; bg: Gaussian width; c: center energy; dc: energetic spacing of an effective progression (note: negative for stimulated emission); hr: Huang-Rhys factor.

\begin{tabular}{|c|c|c|c|c|c|c|}
\hline \multicolumn{7}{|c|}{ State: S in phase: erythrosine, NP } \\
\hline PB & a: -1.000 & bg: 0.205 & c: 2.350 & dc: 0.187 & hr: 0.500 & s: 0.000 \\
\hline SE & a: -1.000 & bg: 0.187 & c: 2.240 & dc: -0.187 & hr: 0.331 & s: 0.000 \\
\hline PAS1 & a: 2.727 & bg: 0.261 & c: 2.703 & dc: 0.187 & hr: 1.611 & s: 0.605 \\
\hline PAS2 & a: 0.079 & bg: 0.150 & c: 3.766 & dc: 0.187 & hr: 0.000 & s: 0.000 \\
\hline \multicolumn{7}{|c|}{ State: EA in phase: erythrosine, NP } \\
\hline $\begin{array}{c}\text { EA } \\
\text { Gauss } \\
\text { linked }\end{array}$ & w1: 0.263 & w2: 0.000 & & & \\
\hline PB & a: -1.000 & bg: 0.200 & c: 2.350 & dc: 0.187 & hr: 0.500 & s: 0.000 \\
\hline \multicolumn{7}{|c|}{ State: in phase: t1, NP } \\
\hline PB & a: -1.000 & bg: 0.211 & c: 3.233 & dc: 0.200 & hr: 1.745 & s: 0.000 \\
\hline PAP1 & a: 1.355 & bg: 0.800 & c: 2.403 & dc: 0.200 & hr: 0.000 & s: 0.000 \\
\hline \multicolumn{7}{|c|}{ State: T in phase: erythrosine, NP } \\
\hline PB & a: -1.000 & bg: 0.133 & c: 2.353 & dc: 0.187 & hr: 0.600 & s: 0.000 \\
\hline PAT1 & a: 0.053 & bg: 0.055 & c: 2.554 & dc: 0.185 & hr: 0.003 & s: 0.240 \\
\hline PAT2 & a: 1.049 & bg: 1.189 & c: 2.411 & dc: 0.185 & hr: 0.010 & s: -0.680 \\
\hline PAT3 & a: 0.413 & bg: 0.407 & c: 3.275 & dc: 0.185 & hr: 0.000 & s: 1.093 \\
\hline PAT4 & a: 0.104 & bg: 0.074 & c: 2.348 & dc: 0.000 & hr: 0.000 & s: 0.000 \\
\hline
\end{tabular}



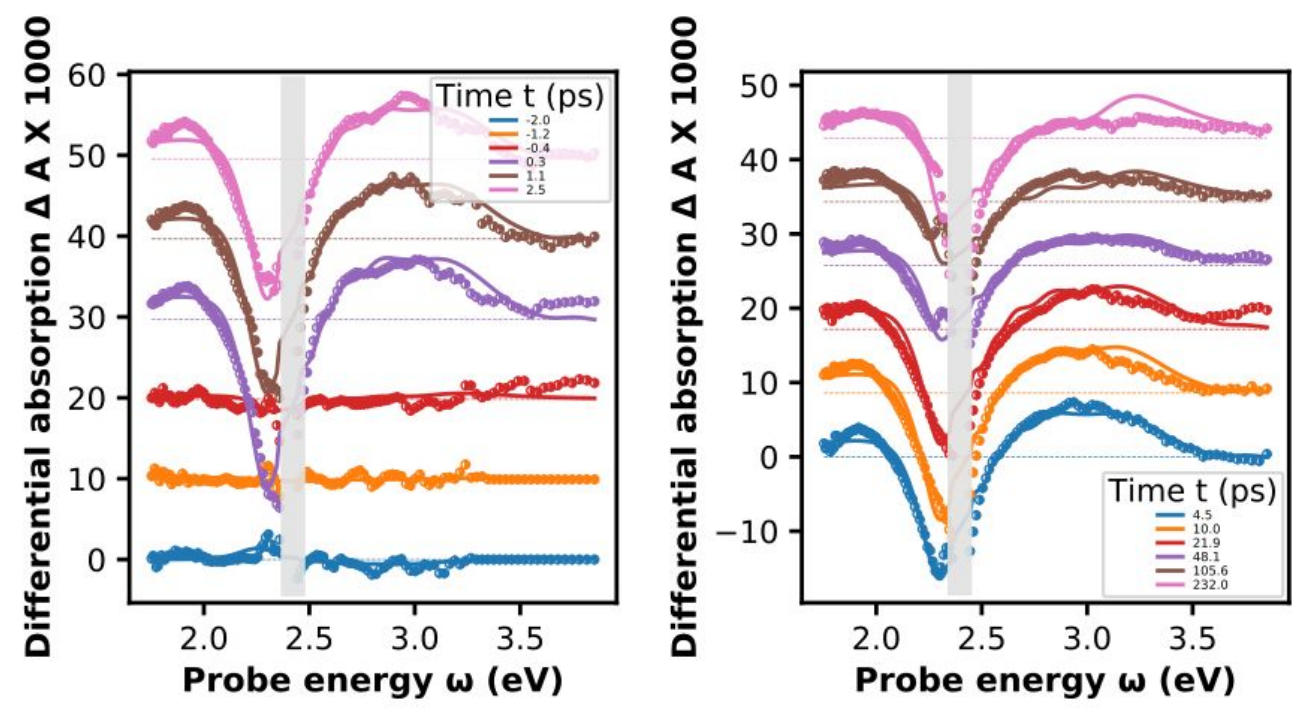

Figure S25. Transient absorption (TA) spectra of SM1:E/l with iodide. Pump energy 2.3 $\mathrm{eV}$ with $150 \mathrm{fs}$ pulses of $450 \mathrm{~nJ}$ pulse energy, at different pump-probe delay times, as given in the inset (symbols). Lines are fits obtained by matrix-multiplying the solid lines in Figure S26 a and Figure S26 b,c, of same color, according to eq. 5. Grey bar indicates region of pump pulse scattering.

a) SVD cross-sections

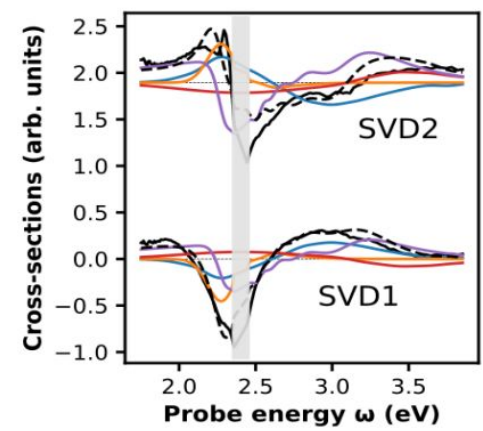

b) Singlet dynamics

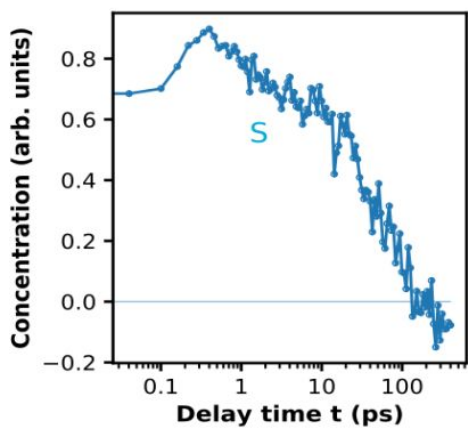

c) Charge and triplet dynamics

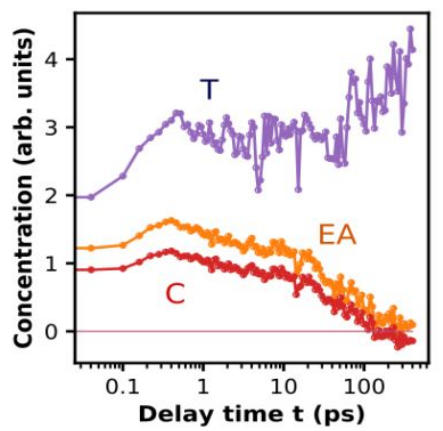

Figure S26. Spectral decomposition of SM1:E/l. (a) Characteristic spectra of a singular value decomposition (SVD) of the time-resolved TA spectrum (symbols in Figure S25) (black solid line) and reconstruction by fitting photophysical model spectra of singlet (blue), electroabsorption (EA) of erythrosin (orange), charges in molecule $\mathbf{1}$ (red) and triplet (green) states (black dashed lines). (b) and (c) Resulting time-resolved concentrations. Grey bar indicates region of pump pulse scattering. 
Figure S25 shows TA spectra for SM1:E/I. At all times, which includes immediately after the pump pulse, we see only PB from $E$ but not from the SM1. This means that the iodide influences the photoexcitation dynamics already during the pump pulse. The TA spectra of Figure S25, especially at long delay times, are similar to those of Figure S20 at long times. Indeed, a fitting of the SVD spectra (Figure S23a) using the same spectral parameters as in the previous experiment results in the erythrosine triplet state being the dominant photoexcitation. The remaining charge population upon recombination creates even more triplets.

Table S3. Spectral model parameters for the states in Figure S26. The significance of the fitting parameters is as follows. a: spectral weight; bg: Gaussian width; c: center energy; dc: energetic spacing of an effective progression (note: negative for stimulated emission)

\begin{tabular}{|c|c|c|c|c|c|c|}
\hline \multicolumn{7}{|c|}{ State: S in phase: erythrosine B, NP } \\
\hline PB & a: -1.000 & bg: 0.205 & c: 2.350 & dc: 0.187 & hr: 0.500 & s: 0.000 \\
\hline SE & a: -1.000 & bg: 0.187 & c: 2.240 & dc: -0.187 & hr: 0.331 & s: 0.000 \\
\hline PAS1 & a: 2.727 & bg: 0.261 & c: 2.703 & dc: 0.187 & hr: 1.611 & s: 0.605 \\
\hline PAS2 & a: 0.079 & bg: 0.150 & c: 3.766 & dc: 0.187 & hr: 0.000 & s: 0.000 \\
\hline \multicolumn{7}{|c|}{ State: EA in phase: erythrosine B, NP } \\
\hline EA & w1: 0.263 & w2: 0.000 & & & \\
\hline PB & a: -1.000 & bg: 0.200 & c: 2.350 & dc: 0.187 & hr: 0.500 & s: 0.000 \\
\hline \multicolumn{7}{|c|}{ State: P in phase: t1, NP } \\
\hline PB & a: -1.000 & bg: 0.211 & c: 3.233 & dc: 0.200 & hr: 1.745 & s: 0.000 \\
\hline PAP1 & a: 1.355 & bg: 0.800 & c: 2.403 & dc: 0.200 & hr: 0.000 & s: 0.000 \\
\hline \multicolumn{7}{|c|}{ State: T in phase: erythrosine B, NP } \\
\hline PB & a: -1.000 & bg: 0.133 & c: 2.353 & dc: 0.187 & hr: 0.600 & s: 0.000 \\
\hline PAT1 & a: 0.053 & bg: 0.061 & c: 2.554 & dc: 0.185 & hr: 0.003 & s: 0.240 \\
\hline PAT2 & a: 1.049 & bg: 1.189 & c: 2.411 & dc: 0.185 & hr: 0.010 & s: -0.680 \\
\hline PAT3 & a: 0.413 & bg: 0.407 & c: 3.275 & dc: 0.185 & hr: 0.000 & s: 1.093 \\
\hline PAT4 & a: 0.104 & bg: 0.074 & c: 2.348 & dc: 0.000 & hr: 0.000 & s: 0.000 \\
\hline
\end{tabular}




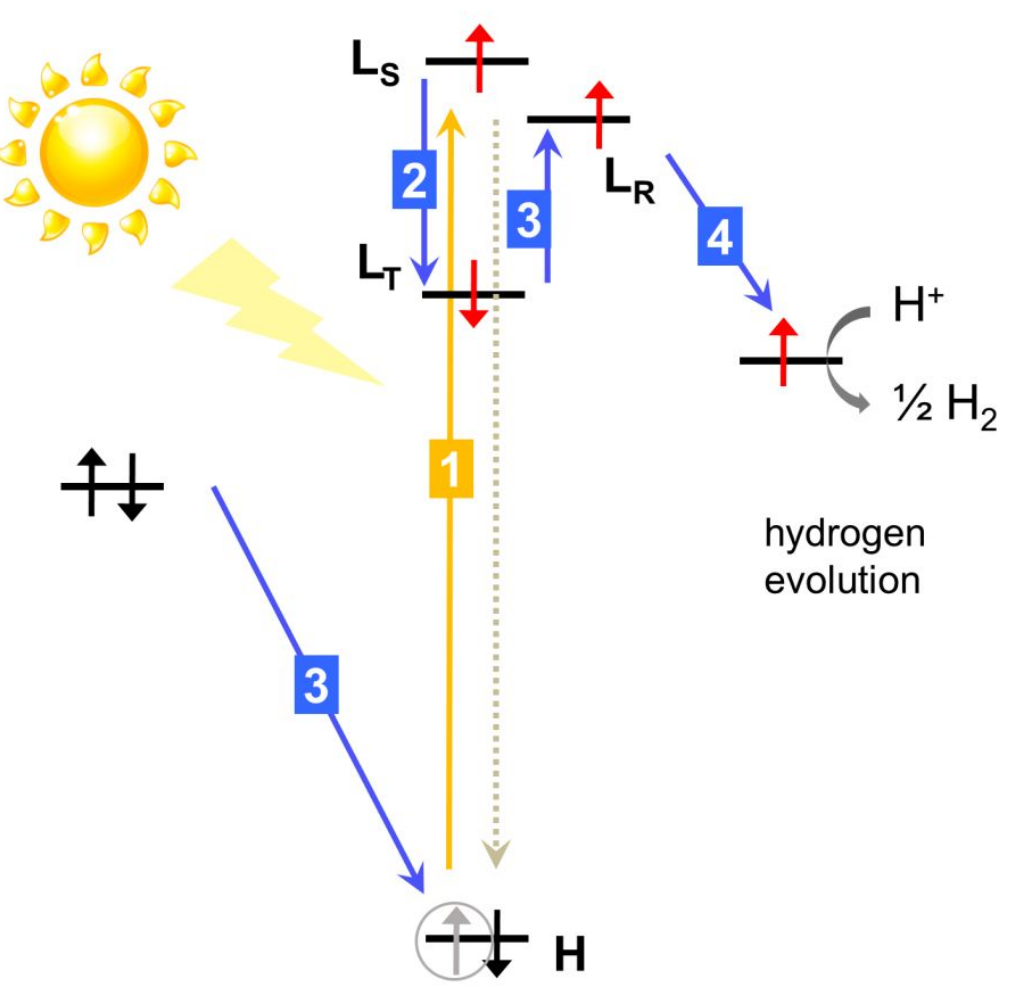

SA

1

Pt

Scheme S1. Proposed photocatalytic cycle of SM1 with and without iodide in a simple one-electron picture. blue arrows with numbers indicate the chronological ordering, undesired loss process are given as grey dashed arrows, $\mathrm{H}$ denoted for homo level, $\mathrm{L}_{s}$ for singlet excited level, $L_{T}$ for triplet excited level, $L_{R}$ for reduced state energy level: After singlet light absorption of 1 to the $S_{1}$ state (1), and fast relaxation to T1 in the presence of iodide (2), an electron is injected by sacrificial agent (SA) (3), reducing $\mathbf{E}$. The electron is transferred from 1 to the platinum co-catalyst (4). 


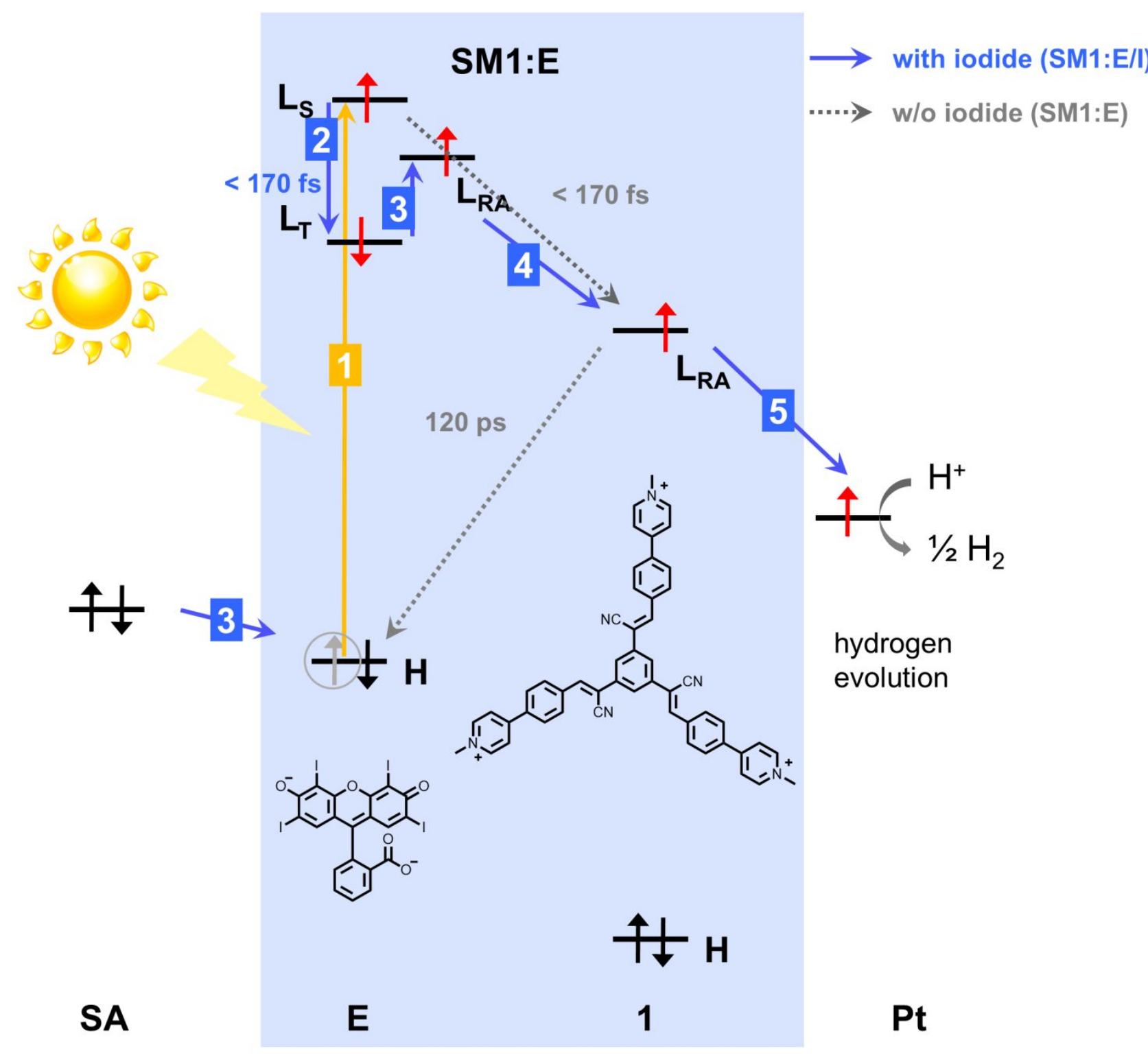

Scheme S2. Proposed photocatalytic cycle of SM1:E with and without iodide in a simple one-electron picture. blue arrows with numbers indicate the chronological ordering, undesired loss process are given as grey dashed arrows, numbers are extracted from the TA studies, $\mathrm{H}$ denoted for homo level, $L_{S}$ for singlet excited level, $L_{T}$ for triplet excited level, $L_{R}$ for reduced state energy level: After singlet light absorption of $\mathbf{E}$ to the $S_{1}$ state (1), and fast relaxation to $T_{1}$ in the presence of iodide (2), an electron is injected by sacrificial agent (SA) (3), reducing $\mathbf{E}$. The electron is transferred from $\mathbf{E}$ to $\mathbf{1}(4)$, followed by the activation of the $\mathrm{Pt}$ co-catalyst (5).

\section{Movie S1.}

Movie of the sample without molecule 1 (prepared from $0.5 \mu \mathrm{mol}$ of $\mathrm{K}_{2} \mathrm{PtCl}_{4}, 0.65 \mu \mathrm{mol}$ of Erythrosine B, $12 \mathrm{mmol}$ of L-ascorbic acid with $0.15 \mathrm{M}$ of Nal in $13 \mathrm{~mL}$ of distilled water) under $300 \mathrm{~W}$ xenon lamp irradiation with $400 \mathrm{~nm}$ long pass filter.

\section{Movie S2.}

Movie of the sample with molecule 1, SM1:E/l (prepared from $0.5 \mu \mathrm{mol}$ of $\mathrm{K}_{2} \mathrm{PtCl}_{4}, 0.65 \mu \mathrm{mol}$ of Erythrosine B and Molecule 1, $12 \mathrm{mmol}$ of L-ascorbic acid with $0.15 \mathrm{M}$ of Nal in $13 \mathrm{~mL}$ of distilled water) under $300 \mathrm{~W}$ xenon lamp irradiation with $400 \mathrm{~nm}$ long pass filter. 


\section{References}

(1) Lee, H.-J.; Kim, H.-J.; Lee, E.-C.; Kim, J.; Park, S. Y. Highly Luminescent and Water-Soluble Two-Dimensional Supramolecular Organic Framework: AllOrganic Photosensitizer Template for Visible-Light-Driven Hydrogen Evolution from Water. Chem. Asian J. 2018, 13, 390-394.

(2) Berndt O.; Bandt, F.; Eichwurzel, I.; Stiel, H. Picosecond Transient Absorption of Xanthene Dyes. Acta Phys. Pol. A. 1999, 95, $207-220$.

(3) Penzkofer, A.; Tyagi, A.; Slyusareva, E.; Sizykh. A. Phosphorescence and Delayed Fluorescence Properties of Fluorine Dyes in Bio-Related Films. Chem. Phys. 2019, 378, 58-65.

(4) Marsh, R. A.; Hodgkiss, J. M.; Albert-Seifried, S.; Friend. R. H. Effect of Annealing on P3HT:PCBM Charge Transfer and Nanoscale Morphology Probed by Ultrafast Spectroscopy. Nano Lett. 2010, 10, 923-930.

(5) Causa, M.; De Jonghe-Risse, J.; Scarongella, M.; Brauer, J. C.; Buchaca-Domingo, E.; Moser, J. -E.; Stingelin, N.; Banerji. N. The Fate of Electron-Hole Pairs in Polymer:Fullerene Blends for Organic Photovoltaic. Nat. Commun. 2016, 7, 12556. 